OPEN ACCESS

The Current-Voltage Characteristics and Partial Pressure Dependence of Defect Controlled Electrochemical Reactions on Mixed Conducting Oxides

To cite this article: Alexander Schmid and Jürgen Fleig 2019 J. Electrochem. Soc. 166 F831

View the article online for updates and enhancements. 


\title{
The Current-Voltage Characteristics and Partial Pressure Dependence of Defect Controlled Electrochemical Reactions on Mixed Conducting Oxides
}

\author{
Alexander Schmid $\oplus^{*, z}$ and Jürgen Fleig \\ Institute of Chemical Technologies and Analytics, Vienna University of Technology, Vienna, A-1060, Austria
}

\begin{abstract}
Electrochemical reactions at solid|gas interfaces of mixed ionic electronic conductors (MIEC), such as oxygen reduction or evolution, differ substantially from usual electrochemical reactions in aqueous solutions. Overpotentials do not directly translate to electrostatic surface potentials but act mainly by changing the concentration of point defects in the MIEC. This has severe consequences for the mechanistic interpretation of current voltage curves of MIEC electrodes. In this contribution it is shown how overpotential dependent defect concentrations affect the current-voltage curves of oxygen reduction and oxygen evolution at MIEC surfaces. Exemplarily, quantitative current-voltage curves are deduced from the known defect chemical data set (Brouwer diagram) of $\mathrm{La}_{0.6} \mathrm{Sr}_{0.4} \mathrm{FeO}_{3-\delta}$ (LSF). Various curve shapes result, from Tafel-like exponential relations to essentially voltage independent limiting currents. Tafel slopes have a very different meaning compared to charge transfer limited reactions at metal electrode interfaces. It is shown how mechanistic information can be obtained from the difference of anodic and cathodic Tafel slopes or by comparing exchange current densities and ac resistances. Moreover, partial pressure dependences of anodic and cathodic currents are deduced, showing that exponents of power laws often do not indicate whether atomic or molecular oxygen species are involved in the rate limiting step. (c) The Author(s) 2019. Published by ECS. This is an open access article distributed under the terms of the Creative Commons Attribution 4.0 License (CC BY, http://creativecommons.org/licenses/by/4.0/), which permits unrestricted reuse of the work in any medium, provided the original work is properly cited. [DOI: 10.1149/2.1031912jes]

(cc) BY
\end{abstract}

Manuscript submitted April 8, 2019; revised manuscript received July 11, 2019. Published August 2, 2019.

Electrochemical reactions at mixed ionic electronic conductor (MIEC)|gas interfaces are highly important for energy conversion and storage. Arguably the most prominent reaction is the oxygen reduction

$$
1 / 2 \mathrm{O}_{2}+2 \mathrm{e}^{-} \longrightarrow \mathrm{O}^{2-}
$$

occurring at solid oxide fuel cell (SOFC) cathodes or, in reverse direction, at solid oxide electrolysis cell (SOEC) anodes. ${ }^{1-8}$ Other important electrochemical solid|gas reactions are water splitting or $\mathrm{CO}_{2}$ electrolysis. ${ }^{9-11}$ The kinetics of the oxygen exchange reactions have been studied intensely on perovskite materials such as $(\mathrm{La}, \mathrm{Sr}) \mathrm{MnO}_{3-\delta} \quad(\mathrm{LSM}),{ }^{12-15}$ (La, Sr $)(\mathrm{Co}, \mathrm{Fe}) \mathrm{O}_{3-\delta} \quad(\mathrm{LSCF}),{ }^{11,16-30}$ $(\mathrm{La}, \mathrm{Sr})(\mathrm{Ti}, \mathrm{Fe}) \mathrm{O}_{3-\delta} \quad(\mathrm{LSTF})^{31-33}$ and $(\mathrm{Ba}, \mathrm{Sr})(\mathrm{Co}, \mathrm{Fe}) \mathrm{O}_{3-\delta}$ (BSCF). ${ }^{34-36}$ Studies aiming at mechanistic information often investigate the exchange current density by means of tracer exchange, ${ }^{37,38}$ conductivity relaxation ${ }^{27}$ or impedance spectroscopy. ${ }^{14,25,39-41}$ While the determination of oxygen exchange rates via such close-toequilibrium methods is well established and frequently employed, it is nevertheless often challenging to extract mechanistic details from such experiments, also because experimental data often include effects of both cathodic and anodic reactions. ${ }^{40,42,43}$ In contrast, experiments far from equilibrium, i.e. at sufficiently high electrode polarization, enhance the reaction rate in one direction while suppressing the reaction in reverse direction, and thus may offer a better view, for example on the dependencies of reaction rates on concentrations. Nevertheless, measurements and mechanistic interpretations of oxygen reduction/evolution current-voltage curves are rather scarce, ${ }^{11,44,45}$ and the concepts for a theoretical description of such current-voltage curves are still under development. ${ }^{46-48}$

Several differences exist between such solid|gas reactions and usual aqueous electrochemical reactions at metal electrodes, and the currentvoltage characteristics of the solid|gas reactions often cannot be described by standard electrochemical models such as Butler-Volmer's equation. ${ }^{47}$ First, in liquid electrochemistry an applied overpotential often translates directly into an electrostatic potential step at the reaction site, i.e. the electrolyte|electrode interface. For electrochemical reactions at MIEC|gas interfaces, however, an applied overpotential acts mainly upon the oxygen chemical potential in the MIEC electrode, and thereby on the MIEC defect concentrations. ${ }^{37,43,49}$ The overpotential might also affect the potential step at the MIEC|gas interface,

*Electrochemical Society Student Member.

${ }^{z}$ E-mail: alex.schmid91@gmail.com but mostly in a rather indirect and complex manner. ${ }^{47}$ Second, the oxygen partial pressure not only determines the concentration of oxygen species adsorbed at the MIEC|gas interface. Rather, the gas phase also affects the oxygen chemical potential in the MIEC electrode and thereby the concentration of defects involved in the reactions. Furthermore, in atmospheres consisting mostly of oxygen and nitrogen it is plausible that charged oxygen adsorbates are the main contributors to a possible electrostatic potential step at the MIEC|gas interface. Therefore, the oxygen partial pressure can also affect the surface potential. ${ }^{47}$

These considerations clearly show that the interpretation of currentvoltage curves and their partial pressure dependencies requires novel concepts beyond standard models known from metal|liquid interfaces. Knowing the defect chemistry of the MIEC electrode and the interrelationship between defect concentrations, oxygen partial pressure and electrode polarization is vital for understanding the kinetics of such reactions. A quantitative approach to tackle such situations has been introduced recently, ${ }^{48}$ and it was shown how the true, i.e. mechanistically meaningful, reaction orders of defects and gas species can be determined from combined voltage and partial pressure variations.

In this contribution, we extend these considerations by modeling current-voltage curves for the known defect chemical data set of a specific material $\left(\mathrm{La}_{0.6} \mathrm{Sr}_{0.4} \mathrm{FeO}_{3-\delta}\right)$ and different possible reaction mechanisms. Various slopes of current-voltage curves can result, depending on the reaction mechanism and partial pressure. However, neither do limiting currents indicate any diffusion limitation, nor do exponential relations reflect charge transfer limitations. The true meanings of Tafel slopes and partial pressure dependent current densities are discussed for specific mechanisms and in general. This opens new ways to draw mechanistic conclusions from empirically measured current-voltage curves and partial pressure dependences.

\section{Rate Equation Model for Defect Controlled Reactions}

In this section we derive an equation that describes the effect of the main experimental parameters (overpotential and oxygen partial pressure) on the current density resulting from oxygen reduction or evolution on a MIEC surface. These considerations are largely based on the rate equation model suggested in Ref. 50 and Ref. 48. It describes the oxygen incorporation and evolution rates in terms of local defect concentrations, surface adsorbates and surface potentials. According to this rate equation model the current densities of anodic oxygen evolution $\left(j_{\mathrm{a}}\right)$ and cathodic oxygen incorporation $\left(j_{\mathrm{c}}\right)$ can be 
expressed as

$$
\begin{aligned}
& j_{\mathrm{a}}=j_{\mathrm{a}}^{0 *} p_{\mathrm{O}_{2}}{ }^{{ }_{\mathrm{p}, \mathrm{a}}} c_{\mathrm{D}, \mathrm{a}}{ }^{{ }_{\mathrm{D}, \mathrm{a}}} \exp \left(\frac{\beta_{\mathrm{a}} e \chi_{0}}{k T}\right) \exp \left(\frac{\beta_{\mathrm{a}} e \Delta \chi}{k T}\right), \\
& j_{\mathrm{c}}=j_{\mathrm{c}}^{0 *} p_{\mathrm{O}_{2}}{ }^{{ }_{\mathrm{p}, \mathrm{c}}} c_{\mathrm{D}, \mathrm{c}}{ }^{{ }_{\mathrm{D}, \mathrm{c}}} \exp \left(\frac{\beta_{\mathrm{c}} e \chi_{0}}{k T}\right) \exp \left(\frac{\beta_{\mathrm{c}} e \Delta \chi}{k T}\right),
\end{aligned}
$$

where the net current density $j$ is the difference between anodic and cathodic current density: $j=j_{\mathrm{a}}-j_{\mathrm{c}}$. Symbols $j_{\mathrm{a}}^{0 *}$ and $j_{\mathrm{c}}^{0 *}$ are constant prefactors including equilibrium constants of equilibria before and after the rate limiting step, $p_{\mathrm{O}_{2}}$ is the oxygen partial pressure and $\nu_{\mathrm{p}, \mathrm{a}}$ and $\nu_{\mathrm{p}, \mathrm{c}}$ are the corresponding reaction orders for the anodic and cathodic reactions. These partial pressure related factors describe the effect of $p_{\mathrm{O}_{2}}$ on the surface adsorbates involved in the rate liming step but do not include the $p_{\mathrm{O}_{2}}$ dependence of electronic or ionic defect concentrations.

Defects are treated separately and symbols $c_{\mathrm{D}, \mathrm{a}}$ and $c_{\mathrm{D}, \mathrm{c}}$ are the concentrations of the defect species in the MIEC being relevant in anodic or cathodic direction, with their corresponding reaction orders $\nu_{\mathrm{D}, \mathrm{a}}$ and $\nu_{\mathrm{D}, \mathrm{c}}$. If multiple defects are relevant, $c_{\mathrm{D}}{ }^{{ }_{\mathrm{D}}}$ has to be expanded to a product such as $c_{\mathrm{D}_{1}}{ }^{{ }^{D_{1}}} c_{\mathrm{D}_{2}}{ }^{{ }^{\mathrm{D}_{2}}}$. These relevant defects are either reaction partners in the rate determining step, or they are involved in preceding or succeeding equilibria. $\beta_{\mathrm{a}}$ and $\beta_{\mathrm{c}}$ are factors depending on the reaction mechanism, $\chi_{0}$ and $\Delta \chi$ are the equilibrium surface potential and its variation upon current flow and $e, k$ and $T$ have their usual meaning of elementary charge, Boltzmann constant and temperature. Please note, the anodic and cathodic current densities in Equation 2 and 3 represent the ionic current flowing in the MIEC from the surface toward the MIEClelectrolyte interface. However, it does not reflect the charge transferred across the MIEC surface itself since this depends on the specific mechanism. In principle, it is even possible to incorporate neutral oxygen and ionize the atoms within the MIEC; then there is no charge flow across the MIEC surface itself.

In equilibrium, i.e. at zero overpotential, the anodic and cathodic current densities are equal, thus

$$
\begin{aligned}
& j_{\mathrm{a}}{ }^{\mathrm{eq}}=j^{0}=j_{\mathrm{a}}^{0 *} p_{\mathrm{O}_{2}}{ }^{{ }_{\mathrm{p}, \mathrm{a}}} c_{\mathrm{D}, \mathrm{a}}^{\mathrm{eq}, \mathrm{a}} \exp \left(\frac{\beta_{\mathrm{a}} e \chi_{0}}{k T}\right), \\
& j_{\mathrm{c}}{ }^{\mathrm{eq}}=j^{0}=j_{\mathrm{c}}^{0 *} p_{\mathrm{O}_{2}}{ }^{{ }_{\mathrm{p}, \mathrm{c}}} c_{\mathrm{D}, \mathrm{c}}^{\mathrm{eq}, \mathrm{v}}{ }^{{ }_{\mathrm{D}} \mathrm{c}} \exp \left(\frac{\beta_{\mathrm{c}} e \chi_{0}}{k T}\right),
\end{aligned}
$$

where $c_{\mathrm{D}, \mathrm{a}}^{\mathrm{eq}}$ and $c_{\mathrm{D}, \mathrm{c}}^{\mathrm{eq}}$ are the equilibrium concentrations of the relevant defects and $j^{0}$ is the exchange current density. This exchange current density is thus determined by the oxygen partial pressure, the equilibrium defect concentrations and the equilibrium surface potential $\chi_{0}$. For a given temperature, the equilibrium defect concentrations are defined by $p_{\mathrm{O}_{2}}$ and the defect chemical equilibrium constant(s) of the MIEC. The equilibrium surface potential $\chi_{0}$ may also depend on $p_{\mathrm{O}_{2}} \cdot{ }^{41,47}$ Thus, for a given material, all variable factors can be traced back to the gas phase and the exchange current density $j^{0}$ depends on $p_{\mathrm{O}_{2}}$ solely. Combining Equations 2 and 3 with Equations 4 and 5 leads to

$$
\begin{aligned}
& j_{\mathrm{a}}=j^{0} \frac{c_{\mathrm{D}, \mathrm{a}}{ }^{{ }_{\mathrm{D}, \mathrm{a}}}}{c_{\mathrm{D}, \mathrm{a}}^{\mathrm{eq}, \mathrm{a}}} \exp \left(\frac{\beta_{\mathrm{a}} e \Delta \chi}{k T}\right), \\
& j_{\mathrm{c}}=j^{0} \frac{c_{\mathrm{D}, \mathrm{c}}{ }^{{ }^{{ }_{\mathrm{D}}, \mathrm{c}}}}{c_{\mathrm{D}, \mathrm{c}}^{\mathrm{eq}}{ }^{{ }^{{ }}{ }^{\mathrm{c}}}} \exp \left(\frac{\beta_{\mathrm{c}} e \Delta \chi}{k T}\right),
\end{aligned}
$$

and the net current density is then

$$
j=j^{0}\left[\exp \left(\frac{\beta_{\mathrm{a}} e \Delta \chi}{k T}\right)\left(\frac{c_{\mathrm{D}, \mathrm{a}}}{c_{\mathrm{D}, \mathrm{a}}^{\mathrm{eq}}}\right)^{v_{\mathrm{D}, \mathrm{a}}}-\exp \left(\frac{\beta_{\mathrm{c}} e \Delta \chi}{k T}\right)\left(\frac{c_{\mathrm{D}, \mathrm{c}}}{c_{\mathrm{D}, \mathrm{c}}^{\mathrm{eq}}}\right)^{v_{\mathrm{D}, \mathrm{c}}}\right] .
$$

The relation between surface potential, oxygen partial pressure and overpotential can be very complex and may also vary with overpotential and $p_{\mathrm{O}_{2}} \cdot{ }^{47}$ Accordingly, $\Delta \mathrm{X}$ is generally unknown which makes general predictions of current-voltage curves difficult. However, XPS studies on $\mathrm{La}_{0.6} \mathrm{Sr}_{0.4} \mathrm{FeO}_{3-\delta}$ suggest that the surface potential does not change much with overpotential or $p_{\mathrm{O}_{2}}{ }^{23}$ In the following we therefore assume such a simplified situation with a constant $\chi=\chi_{0}$ and thus $\Delta \chi=0$ for all overpotentials and partial pressures. For such "defect controlled reactions", Equation 8 can be simplified to

$$
j=j^{0}\left[\left(\frac{c_{\mathrm{D}, \mathrm{a}}}{c_{\mathrm{D}, \mathrm{a}}^{\mathrm{eq}}}\right)^{v_{\mathrm{D}, \mathrm{a}}}-\left(\frac{c_{\mathrm{D}, \mathrm{c}}}{c_{\mathrm{D}, \mathrm{c}}^{\mathrm{eq}}}\right)^{v_{\mathrm{D}, \mathrm{c}}}\right],
$$

and for the exchange current densities (Equations 4 and 5) we get

$$
\begin{aligned}
& j^{0}=j_{\mathrm{a}}^{0} p_{\mathrm{O}_{2}}{ }^{{ }_{\mathrm{p}, \mathrm{a}}} c_{\mathrm{D}, \mathrm{a}}^{\mathrm{eq}, \mathrm{v}, \mathrm{a}}, \\
& j^{0}=j_{\mathrm{c}}^{0} p_{\mathrm{O}_{2}}{ }^{{ }{ }_{\mathrm{p}, \mathrm{c}}} c_{\mathrm{D}, \mathrm{c}}^{\mathrm{eq}}{ }^{{ }_{\mathrm{D}, \mathrm{c}}} .
\end{aligned}
$$

where the prefactors $j_{\mathrm{a}}^{0}$ and $j_{\mathrm{c}}^{0}$ include $j_{\mathrm{a}}^{0 *}$ and $j_{\mathrm{c}}^{0 *}$ but also include the $p_{\mathrm{O}_{2}}$ independent equilibrium surface potential factors $\exp \left(\frac{\beta e \chi_{0}}{k T}\right)$.

An applied overpotential causes a net current density $(j \neq 0)$ by driving the defect concentrations in the MIEC electrode away from their equilibrium values, i.e. by changing the ratio $c_{\mathrm{D}} / c_{\mathrm{D}}^{\mathrm{eq}}$. (Please note: If several defects are involved in anodic or cathodic direction these ratios have to be replaced by products with ratios for each relevant ionic or electronic defect.) The current-voltage characteristics is therefore a direct consequence of the relation between defect concentrations and electrode overpotential. This relation can be quantified in the following manner: If the ion and electron transport in the electrode is much faster than the surface exchange reaction, the electrode overpotential $\eta$ directly translates to a change in (molecular) oxygen chemical potential $\mu_{\mathrm{O}_{2}}$ in the MIEC according to the Nernstian relation ${ }^{37,49}$

$$
\mu_{\mathrm{O}_{2}}=4 e \eta+k T \ln \left(\frac{p_{\mathrm{O}_{2}}}{1 \mathrm{bar}}\right) .
$$

Under polarization, the defects are thus no longer in equilibrium with the gas phase $\left(p_{\mathrm{O}_{2}}\right)$, but are defined by the oxygen chemical potential in the electrode film according to Equation 12. Accordingly, the change in oxygen chemical potential by $4 \mathrm{e} \eta$ causes a change in defect concentrations and thus a current according to Equation 9.

The (equilibrium) defect chemistry of MIEC oxides is often described by Brouwer diagrams, which relate the defect concentrations to the oxygen partial pressure. ${ }^{51}$ Actually, this $p_{\mathrm{O}_{2}}$ dependence of defect concentrations in the MIEC is a $\mu_{\mathrm{O}_{2}}$ dependence. Only in equilibrium, $\mu_{\mathrm{O}_{2}}$ in the MIEC is given by $\mu_{\mathrm{O}_{2}}=k T \ln \left(\frac{p_{\mathrm{O}_{2}}}{1 \mathrm{bar}}\right)$, while upon a current the overpotential changes $\mu_{\mathrm{O}_{2}}$ according to Equation 12. Studies of the chemical capacitance of LSF confirmed the equivalence of $\mu_{\mathrm{O}_{2}}$ changes by electrode overpotential and gas phase $p_{\mathrm{O}_{2}}{ }^{49} \mathrm{In}$ simplified Brouwer diagrams the specific $c_{\mathrm{D}}$ vs. $\mu_{\mathrm{O}_{2}}$ relations switch between two extremes: either the defect concentrations are essentially $\mu_{\mathrm{O}_{2}}$ independent, or there exists an exponential relation ${ }^{51}$

$$
c_{\mathrm{D}}=c_{\mathrm{D}}^{\prime} \exp \left(n \frac{\mu_{\mathrm{O}_{2}}-\mu_{\mathrm{O}_{2}}^{\prime}}{k T}\right) \text {. }
$$

For equilibrium situations $(\eta=0)$ the latter is equivalent to the power law relation

$$
c_{\mathrm{D}}=c_{\mathrm{D}}^{\prime}\left(\frac{p_{\mathrm{O}_{2}}}{p_{\mathrm{O}_{2}}^{\prime}}\right)^{n}
$$

between defect concentrations for two oxygen partial pressures $p_{\mathrm{O}_{2}}$ and $p_{\mathrm{O}_{2}}^{\prime}$. Exponent $n$ describes the slope in the Brouwer diagram, e.g. 0.25 for electron holes as minority charge carriers in an acceptor doped MIEC or -0.5 for oxygen vacancies as minority defects.

Upon a current and for $c_{\mathrm{D}}^{\prime}=c_{\mathrm{D}}^{\text {eq }}$ we can combine Equations 12 and 13 and the resulting relation between defect concentration and the overpotential $\eta$ is

$$
c_{\mathrm{D}}=c_{\mathrm{D}}^{\mathrm{eq}} \exp \left(\frac{4 e n \eta}{k T}\right) .
$$

Combining Equations 9 and 15 finally yields

$$
j=j^{0}\left[\exp \left(\frac{4 e n_{\mathrm{D}, \mathrm{a}} \nu_{\mathrm{D}, \mathrm{a}} \eta}{k T}\right)-\exp \left(\frac{4 e n_{\mathrm{D}, \mathrm{c}} \nu_{\mathrm{D}, \mathrm{c}} \eta}{k T}\right)\right] .
$$


Again, involvement of several defect species in anodic or cathodic direction would mean that $n_{\mathrm{D}, \mathrm{a}} \nu_{\mathrm{D}, \mathrm{a}}$ or $n_{\mathrm{D}, \mathrm{c}} \nu_{\mathrm{D}, \mathrm{c}}$ have to be expanded to products including several defects, e.g. $\prod_{i} n_{\mathrm{D}, \mathrm{a}, i} v_{\mathrm{D}, \mathrm{a}, i}$ or $\prod_{i} n_{\mathrm{D}, \mathrm{c}, i} v_{\mathrm{D}, \mathrm{c}, i}$. Equation 16 shows that for relevant defects following exponential relations with respect to $\mu_{\mathrm{O}_{2}}$, defect controlled reactions can also exhibit Butler-Volmer like exponential current-voltage characteristics. However, the reason for this curve shape is different than for Butler-Volmer reaction kinetics in aqueous systems. There, the two exponential terms originate from rate limiting charge transfer affected by a voltage induced change of the interfacial Galvani potential and thus of activation energies or shifts of electronic energy levels in solution.$^{52}$ In our MIEC case, on the other hand, the exponential curve shape is a consequence of changes in defect concentrations by the applied voltage, i.e. of Nernst's equation. The Tafel slopes of the exponential curves thus no longer include a symmetry factor of a charge transfer process. Rather, the slope is given by a product of the $\mu_{\mathrm{O}_{2}}$ dependence of the relevant defects $\left(n_{\mathrm{D}, \mathrm{a}}\right.$ and $\left.n_{\mathrm{D}, \mathrm{c}}\right)$, the defect reaction orders of the specific reaction mechanism $\left(v_{\mathrm{D}, \mathrm{a}}\right.$ and $\left.\nu_{\mathrm{D}, \mathrm{c}}\right)$ and the factor of $4 e$ from the relation between $\mu_{\mathrm{O}_{2}}$ and $\eta$ in Nernst's Equation 12.

For the sake of comparison, we may write Equation 16 formally equivalent to a Butler-Volmer equation, i.e. as

$$
j=j^{0}\left[\exp \left(\frac{m_{\mathrm{a}} \alpha e \eta}{k T}\right)-\exp \left(-\frac{m_{\mathrm{c}}(1-\alpha) e \eta}{k T}\right)\right],
$$

and for $\alpha=\frac{1}{2}$ we thus get

$$
\begin{gathered}
m_{\mathrm{a}}=8 n_{\mathrm{D}, \mathrm{a}} \nu_{\mathrm{D}, \mathrm{a}} \\
m_{\mathrm{c}}=-8 n_{\mathrm{D}, \mathrm{c}} \nu_{\mathrm{D}, \mathrm{c}} .
\end{gathered}
$$

Hence, the factor $m_{\mathrm{a}, \mathrm{c}}$, which may indicate the number of electrons transferred ${ }^{52}$ in standard liquid electrochemical cases, has a very different meaning here. As an example: for two oxygen vacancies being involved in the reaction rate in cathodic direction $\left(v_{D, c}=2\right)$ and a vacancy related slope of -0.5 in a Brouwer diagram we find a $m_{\mathrm{c}}$ value of 8 . If, on the other hand, the relevant defect concentrations are independent of the oxygen chemical potential $\left(v_{D}=0\right)$, the corresponding current density becomes $\eta$-independent, thus pretending a kind of diffusion limitation in a standard interpretations. In the following we will exemplify these relations between defect concentrations and current-voltage characteristics for different mechanisms on the perovskite material $\mathrm{La}_{0.6} \mathrm{Sr}_{0.4} \mathrm{FeO}_{3-\delta}$ (LSF).

\section{LSF Defect Model}

In order to specify defect controlled current-voltage characteristics of a mixed conducting material, knowledge on its defect chemistry is required. For LSF as a model material the defect chemical model is well established and includes oxygen vacancies $\left(\mathrm{V}_{\mathrm{O}}\right)$, electron holes $\left(h^{*}\right)$ and electrons $\left(e^{\prime}\right)$ as the main point defects. Electrons and holes are frequently attributed to the Fe cations and thus holes correspond to $\mathrm{Fe}^{4+}\left(\mathrm{Fe}_{\mathrm{Fe}}^{\cdot}\right)$ while electrons are realized by $\mathrm{Fe}^{2+}\left(\mathrm{Fe}_{\mathrm{Fe}}^{\prime}\right)$. Some recent studies suggest that holes are more distributed across the oxygen ions surrounding the corresponding Fe ion. ${ }^{53-55}$ In this paper we use the general notation $\left(h^{*}\right.$ and $\left.\mathrm{e}^{\prime}\right)$. Thus the following two defect chemical equilibria exist

$$
\begin{gathered}
1 / 2 \mathrm{O}_{2}+\mathrm{V}_{\mathrm{O}}^{\ddot{*}}+2 \mathrm{Fe}_{\mathrm{Fe}}^{\times} \rightleftharpoons \mathrm{O}_{\mathrm{O}}^{\times}+2 \mathrm{~h}^{\circ} \\
2 \mathrm{Fe}_{\mathrm{Fe}}^{\times} \rightleftharpoons \mathrm{h}^{\cdot}+\mathrm{e}^{\prime}
\end{gathered}
$$

with mass action constants

$$
\begin{gathered}
K_{\mathrm{ox}}=\exp \left(\frac{T \Delta S_{\mathrm{ox}}-\Delta H_{\mathrm{ox}}}{R T}\right)=\frac{c_{\mathrm{Ox}} c_{\mathrm{h}}{ }^{2}}{\sqrt{p_{\mathrm{O}_{2}}} c_{\mathrm{V}} c_{\mathrm{Fe}}{ }^{2}} \\
K_{\mathrm{i}}=\exp \left(\frac{T \Delta S_{\mathrm{i}}-\Delta H_{\mathrm{i}}}{R T}\right)=\frac{c_{\mathrm{h}} c_{\mathrm{e}}}{c_{\mathrm{Fe}}{ }^{2}},
\end{gathered}
$$

where $c_{\mathrm{V}}, c_{\mathrm{h}}, c_{\mathrm{e}}, c_{\mathrm{Fe}}, c_{\mathrm{Ox}}$ are the concentrations of oxygen vacancies, holes, electrons, $\mathrm{Fe}^{3+}\left(\mathrm{Fe}_{\mathrm{Fe}}^{\times}\right)$and oxide ions, respectively. When dealing with dilute defects, the concentration of regular units is much

\section{Table I. Enthalpies and entropies for oxygen exchange $\left(\Delta H_{0 x}\right.$,} $\left.\Delta S_{\mathrm{ox}}\right)$ and electron/hole pair formation $\left(\Delta H_{\mathrm{i}}, \Delta S_{\mathrm{i}}\right){ }^{57}$

$$
\begin{gathered}
\Delta H_{\mathrm{ox}}\left(\mathrm{kJmol}^{-1}\right) \\
\Delta S_{\mathrm{ox}}\left(\mathrm{Jmol}^{-1} \mathrm{~K}^{-1}\right) \\
\Delta H_{\mathrm{i}}\left(\mathrm{kJmol}^{-1}\right) \\
\Delta S_{\mathrm{i}}\left(\mathrm{Jmol}^{-1} \mathrm{~K}^{-1}\right)
\end{gathered}
$$$$
\begin{aligned}
-95.62 & \pm 4.18 \\
-54.27 & \pm 4.43 \\
95.75 & \pm 2.05 \\
-21.63 & \pm 2.13
\end{aligned}
$$

larger than the defect concentrations and does not change notably with $p_{\mathrm{O}_{2}}$ or overpotential, it can be included in the respective equilibrium constants. In heavily doped mixed conductors such as LSF though, the concentration of ideal lattice species can easily be in the same range as defect concentrations. Therefore $\mathrm{Fe}_{\mathrm{Fe}}^{\times}$and $\mathrm{O}_{\mathrm{O}}^{\times}$are also included in the defect chemical equations and take into account the fact of site restriction. Activity coefficients due to defect interaction, however, are not included. ${ }^{56-59}$

Enthalpies and entropies for the oxygen exchange reaction and electron/hole pair formation in macroscopic LSF bulk samples were determined by thermogravimetry and coulometric titration. ${ }^{57,58} \mathrm{Ta}-$ ble I shows enthalpies and entropies for bulk LSF. Our own measurements of the chemical capacitance of thin LSF films (30 to $120 \mathrm{~nm}$ ) are in good agreement for the oxygen exchange, but indicate higher electronic charge carrier concentrations in thin films. ${ }^{49}$ For the following calculations we use the established bulk thermodynamic data set.

The Brouwer diagram for these bulk data ${ }^{57}$ is displayed in Figure 1 for $600{ }^{\circ} \mathrm{C}$. The ideal lattice sites $\left(\mathrm{O}_{\mathrm{O}}^{\times}\right.$and $\left.\mathrm{Fe}_{\mathrm{Fe}}^{\times}\right)$are included to show that, especially for iron $\left(\mathrm{Fe}_{\mathrm{Fe}}^{\times}\right)$and electron holes $\left(\mathrm{h}^{\cdot}\right)$ in the oxidizing regime, the concentrations can become comparable. For example, in high oxygen partial pressure the concentration of electron holes is 0.4 per unit cell, and thus the concentration of ideal lattice iron is 0.6 per unit cell. However, the voltage driven relative concentration changes of those ideal lattice species are small compared to those of the minority point defects, and thus their impact on the current-voltage characteristics is rather small.



Figure 1. Brouwer diagram for LSF based on bulk data. ${ }^{57}$ Slope markers above the respective curves denote the power law exponent in the defect concentration vs. $p_{\mathrm{O}_{2}}$ relation, see Equation 14. Slope markers below the respective curves indicate the exponent in the defect concentration vs. $\mu_{\mathrm{O}_{2}}$ relation, see Equation 13 
Please note that these data reflect the defect chemistry of the LSF bulk. The surface defect chemistry relevant for the oxygen exchange reactions is not necessarily the same as the defect chemistry in the LSF bulk. However, we may assume that the surface defect chemistry is still governed by the same defect chemical equilibria, though with different equilibrium constants. Accordingly, the surface Brouwer diagram differs from the bulk diagram primarily by a shift on the $p_{\mathrm{O}_{2}}$ axis and a change in the electronic charge carrier concentrations. Hence, even though the following calculations are based on bulk defect chemistry they are also valid for the (unknown) surface defect chemistry, though the $p_{\mathrm{O}_{2}}$ validity ranges are most probably shifted. In the next sections we combine the kinetic model of above and the defect model of LSF and derive current-voltage curves for different specific reaction mechanisms, atomic and molecular ones, i.e. with an atomic or a molecular oxygen species in the rate determining step. Please note: The two reaction mechanisms discussed below were chosen to illustrate our "defect controlled" reaction kinetics model and its predictions, rather than with regards to their likeliness. They are representative also for more complex (and thus more realistic) mechanisms.

\section{Current-Voltage Curves for an Atomic Mechanism}

General equations and exchange current density.-First we want to examine a simple atomic mechanism, i.e. one where the oxygen species in the rate determining step is atomic. This mechanism further illustrates the approach described above and also allows simple interpretation of the resulting dependencies. We assume fast adsorption and dissociation of oxygen 24 , followed by the rate determining incorporation of the adsorbed $\mathrm{O}$ ad-atom 25 , and a fast reduction to form oxide ions 26 .

$$
\begin{aligned}
& 1 / 2 \mathrm{O}_{2} \rightleftharpoons \mathrm{O}_{(\mathrm{ad})} \\
& \mathrm{O}_{(\mathrm{ad})}+\mathrm{V}_{\mathrm{O}} \longleftrightarrow \mathrm{O}_{\mathrm{O}}
\end{aligned}
$$



Thus,

$$
\begin{gathered}
j_{\mathrm{a}}=k_{\mathrm{a}} c_{\mathrm{Opp}} \\
j_{\mathrm{c}}=k_{\mathrm{c}} c_{\mathrm{V}} \theta_{\mathrm{at}}^{0},
\end{gathered}
$$

where $c_{\mathrm{Opp}}$ is the concentration of lattice oxygen with two positive relative charges $\left(\mathrm{O}_{\mathrm{O}}^{*}\right)$ and $\theta_{\mathrm{at}}^{0}$ is the surface coverage with neutral atomic oxygen $\left(\mathrm{O}_{(\mathrm{ad})}\right)$. The constant factors $k_{\mathrm{a}}$ and $k_{\mathrm{c}}$ are proportional to the rate constants of the anodic and cathodic reactions. The fast steps 26 and 24 are always in equilibrium and thus can be described by the mass action laws

$$
\begin{gathered}
K_{\mathrm{ion}}=\frac{c_{\mathrm{Ox}} c_{\mathrm{h}}^{2}}{c_{\mathrm{Opp}} c_{\mathrm{Fe}}^{2}} \\
K_{\mathrm{ads}}=\frac{\theta_{\mathrm{at}}^{0}}{\sqrt{p_{\mathrm{O}_{2}}}} .
\end{gathered}
$$

Combing 27 and 28 with 29 and 30 gives

$$
\begin{gathered}
j_{\mathrm{a}}=j_{\mathrm{a}}^{0} c_{\mathrm{h}}{ }^{2} c_{\mathrm{Ox}} c_{\mathrm{Fe}}{ }^{-2} \\
j_{\mathrm{c}}=j_{\mathrm{c}}^{0} \sqrt{p_{\mathrm{O}_{2}}} c_{\mathrm{V}},
\end{gathered}
$$

where $j_{\mathrm{a}}^{0}=k_{\mathrm{a}} / K_{\text {ion }}$ and $j_{\mathrm{c}}^{0}=k_{\mathrm{c}} K_{\text {ads }}$. Thus, Equation 9 becomes:

$$
j=j^{0}\left[\left(\frac{c_{\mathrm{h}}}{c_{\mathrm{h}}^{\mathrm{eq}}}\right)^{2}\left(\frac{c_{\mathrm{Ox}}}{c_{\mathrm{Ox}}^{\mathrm{eq}}}\right)\left(\frac{c_{\mathrm{Fe}}}{c_{\mathrm{Fe}}^{\mathrm{eq}}}\right)^{-2}-\left(\frac{c_{\mathrm{V}}}{c_{\mathrm{V}}^{\mathrm{eq}}}\right)\right] \text {. }
$$

This is a first case where at least in anodic direction several defects are relevant and thus a product of defect terms has to be used in Equation 9. However, the following calculations illustrate that only the $c_{\mathrm{h}}$ factor plays a decisive role for anodic currents.

The exchange current density in Equation 33 is

$$
j^{0}=j_{\mathrm{a}}^{0}\left(c_{\mathrm{h}}^{\mathrm{eq}}\right)^{2} c_{\mathrm{Ox}}^{\mathrm{eq}}\left(c_{\mathrm{Fe}}^{\mathrm{eq}}\right)^{-2}=j_{\mathrm{c}}^{0} \sqrt{p_{\mathrm{O}_{2}}} c_{\mathrm{V}}^{\mathrm{eq}} .
$$

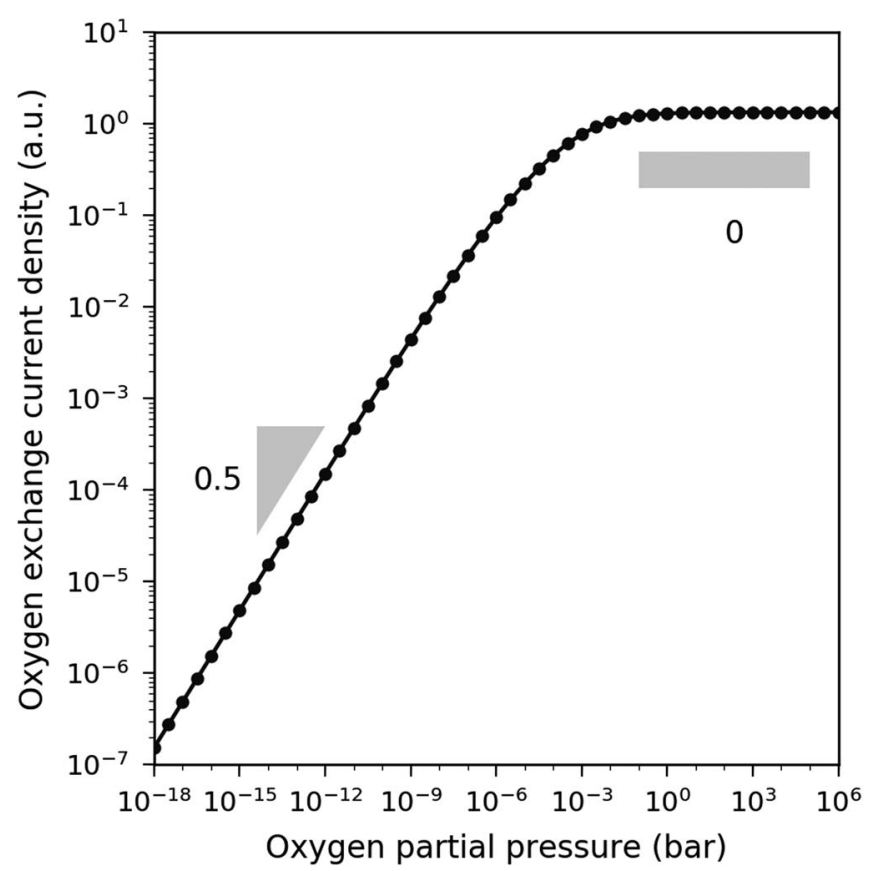

Figure 2. Oxygen exchange current density for the atomic mechanism as a function of oxygen partial pressure, calculated by Equation 34 .

Symbols $c_{\mathrm{h}}^{\mathrm{eq}}, c_{\mathrm{V}}^{\mathrm{eq}}, c_{\mathrm{Fe}}^{\mathrm{eq}}$ and $c_{\mathrm{Ox}}^{\mathrm{eq}}$ denote the equilibrium concentrations (i.e. without current flow) of electron holes, oxygen vacancies, lattice iron and lattice oxide, respectively. The exchange current density $j^{0}$ and the equilibrium defect concentrations are determined by the oxygen partial pressure via the defect chemical equilibria of LSF, i.e. by its Brouwer diagram, see Figure 1.

Knowing the relation between defect concentrations and oxygen partial pressure, we can calculate the $p_{\mathrm{O}_{2}}$ dependence of the exchange current density (Figure 2). The exchange current density is constant at high oxygen partial pressure. Only at lower oxygen partial pressure it scales with $\sqrt{p_{\mathrm{O}_{2}}}$ as one might expect for an atomic mechanism. This is due to the fact that the $p_{\mathrm{O}_{2}}$ dependence of the exchange current density does not only depend on the nature of the oxygen species in the rate determining step, but includes also the reaction orders of the relevant defects (Equation 34) and thus the partial pressure dependence of these defects. In this particular case, the slope of 0 in the high $p_{\mathrm{O}_{2}}$ regime of Figure 2 is a combination of the slope of 0.5 (due to the atomic nature of the mechanism) and a slope of -0.5 due to the oxygen vacancies decreasing with $\sqrt{p_{\mathrm{O}_{2}}}$ in this regime.

An overpotential drives the defect concentration out of equilibrium by changing the oxygen chemical potential, see Equation 12 . The current-voltage curves can be calculated from Equations 33, 12 and the Brouwer diagram (Figure 1) and depend on the oxygen partial pressure in the gas phase. Examples are shown in Figure 3 for a broad $p_{\mathrm{O}_{2}}$ range. These current-voltage characteristics show a variety of Tafel-like exponential regions and current limited regions, both with various $p_{\mathrm{O}_{2}}$ dependencies. In the following, we discuss how these current-voltage characteristics for the anodic oxygen evolution and the cathodic oxygen incorporation are a direct consequence of the defect chemistry of LSF. Please note that related mechanisms, e.g. including formation of one electron hole into the rds (Equation 25), would lead to almost identical curves.

Anodic branch.-In high oxygen partial pressures (Figures 3a and $3 b$ ), the anodic current first increases steeply with overpotential but reaches a plateau already at very low overpotentials. This is because for high $\mu_{\mathrm{O}_{2}}$ the concentration of electron holes is essentially constant, and thus the overpotential does not drive the hole concentration away from its equilibrium value noticeably. Therefore, the only effect of the 
a) Very high $p_{\mathrm{O}_{2}}$



c) Medium $p_{\mathrm{O}_{2}}$

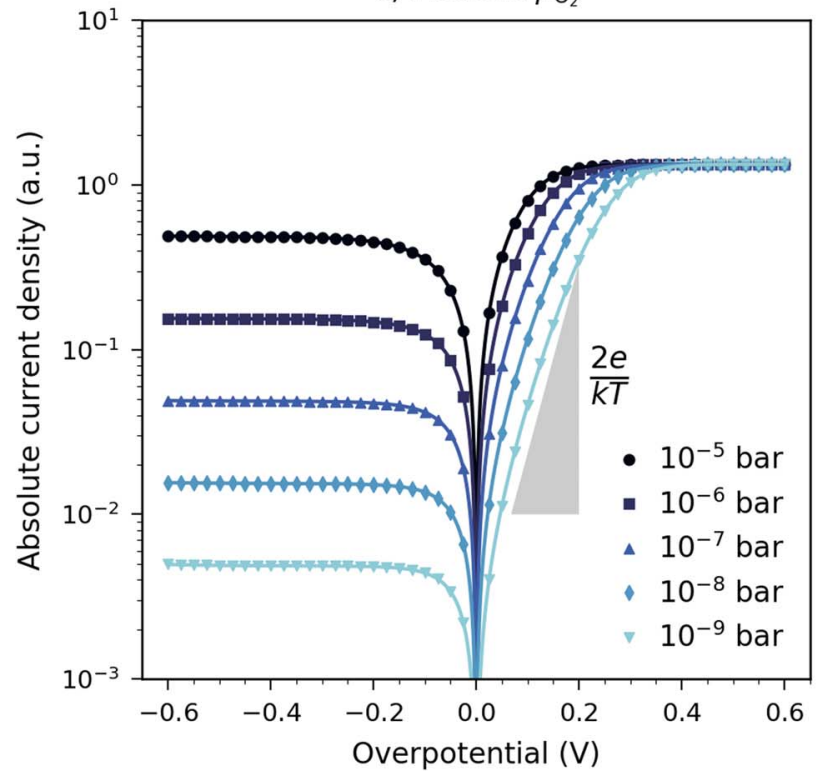

b) High $p_{\mathrm{O}_{2}}$



d) Low $p_{\mathrm{O}_{2}}$

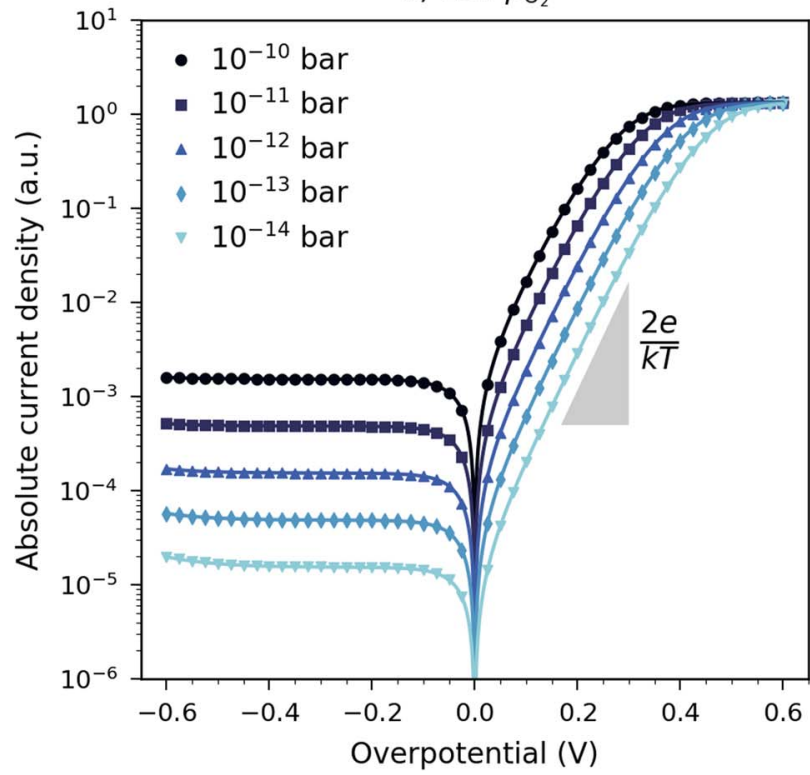

Figure 3. Current voltage characteristics for the atomic mechanism, calculated by Equation 33, for different oxygen partial pressures. Defect concentrations are based on the Brouwer diagram in Figure 1.

overpotential in Equation 33 is to suppress the cathodic current and this is the origin of the first steep increase in current. In the plateau regime, the anodic current-density is then just the exchange current density.

At lower oxygen partial pressures (Figures $3 \mathrm{c}$ and 3d), higher overpotentials are required to reach this current limit, since the lower $\mu_{\mathrm{O}_{2}}$ of the atmosphere has to be compensated by a larger overpotential to drive the electrode to a $\mu_{\mathrm{O}_{2}}$ range of constant hole concentration. For low $p_{\mathrm{O}_{2}}$ and only moderate overpotentials the current-voltage curves thus become exponential, which reflects the exponential relation between electron holes and oxygen chemical potential in this regime. The Tafel slope of those current-voltage curves is $\frac{2 e}{k T}$ and this value consists of three factors:

$$
\frac{\mathrm{d} \ln j_{\mathrm{a}}}{\mathrm{d} \eta}=\frac{\mathrm{d} \ln j_{\mathrm{a}}}{\mathrm{d} \ln c_{\mathrm{h}}} \frac{\mathrm{d} \ln c_{\mathrm{h}}}{\mathrm{d} \mu_{\mathrm{O}_{2}}} \frac{\mathrm{d} \mu_{\mathrm{O}_{2}}}{\mathrm{~d} \eta}=2 \frac{1}{4 k T} 4 e=\frac{2 e}{k T} .
$$

The first factor $\frac{\mathrm{d} \ln j_{\mathrm{a}}}{\mathrm{d} \ln c_{\mathrm{h}}}$ is the reaction order with respect to electron holes, i.e. 2 for this specific mechanism (cf. $v_{D, a}$ in Equation 16), the second one $\frac{\mathrm{d} \ln c_{\mathrm{h}}}{\mathrm{d} \mu_{\mathrm{O}_{2}}}$ is a consequence of the Brouwer diagram (cf. $n_{\mathrm{D}, \mathrm{a}} / k T$ in Equation 16) and the third one comes from Nernst's Equation 12. In this exponential regime, the oxygen evolution current scales with oxygen partial pressure. This surprising accelerating effect of gaseous oxygen on the evolution rate $\left(\mathrm{O}_{2}\right.$ is a reaction product $)$ is only indirect and caused by the LSF defect chemistry. More specific, the (equilibrium) hole concentration increases with $p_{\mathrm{O}_{2}}{ }^{0.25}$, and due to $j^{0}$ being proportional to $\left(c_{\mathrm{h}}^{\mathrm{eq}}\right)^{2}$ (Equation 34 ) the current density for a given overpotential increases with $p_{\mathrm{O}_{2}}{ }^{0.5}$. In the high overpotential range where the hole concentration is constant at 0.4 , however, the effect of $p_{\mathrm{O}_{2}}$ vanishes.

Cathodic branch.-In very high oxygen partial pressures and under moderate cathodic polarization (see Figures $3 \mathrm{a}$ and $3 \mathrm{~b}$ ) the current 
is independent of $p_{\mathrm{O}_{2}}$ and increases exponentially with overpotential; the Tafel-slope in this regime is $-\frac{2 e}{k T}$. At more cathodic overpotentials the current reaches a plateau and in this plateau regime the current becomes $p_{\mathrm{O}_{2}}$ dependent and scales with $\sqrt{p_{\mathrm{O}_{2}}}$. The overpotential where this transition from exponential to flat current-voltage curves occurs also depends on the oxygen partial pressure. Again, this behavior is a direct consequence of the LSF defect chemistry and the relation between defect concentrations and oxygen chemical potential. At high $p_{\mathrm{O}_{2}}$ the oxygen vacancy concentration increases with decreasing $\mu_{\mathrm{O}_{2}}$, i.e. the cathodic overpotential increases the current by driving the oxygen vacancy concentration away from its equilibrium value. The slope of $-\frac{2 e}{k T}$ again consists of three factors, one from the specific mechanism, one from the Brouwer diagram and one from Nernst's equation:

$$
\frac{\mathrm{d} \ln j_{\mathrm{c}}}{\mathrm{d} \eta}=\frac{\mathrm{d} \ln j_{\mathrm{c}}}{\mathrm{d} \ln c_{\mathrm{V}}} \frac{\mathrm{d} \ln c_{\mathrm{V}}}{\mathrm{d} \mu_{\mathrm{O}_{2}}} \frac{\mathrm{d} \mu_{\mathrm{O}_{2}}}{\mathrm{~d} \eta}=1 \frac{-1}{2 k T} 4 e=-\frac{2 e}{k T} .
$$

In this exponential regime the current is $p_{\mathrm{O}_{2}}$ independent because the accelerating effect of $p_{\mathrm{O}_{2}}$ via adsorbates is counter-balanced by the oxygen vacancy concentration decreasing with $\sqrt{p_{\mathrm{O}_{2}}}$.

At very cathodic overpotentials, the oxygen vacancy concentration becomes $\mu_{\mathrm{O}_{2}}$ independent. Thus the cathodic overpotential causes no further increase in oxygen vacancy concentration and the current becomes constant. However, in this regime the accelerating effect of $p_{\mathrm{O}_{2}}$ via adsorbates is no longer counter-balanced by defect changes and therefore the current for a given overpotential scales with $\sqrt{p_{\mathrm{O}_{2}}}$. In higher oxygen partial pressures (higher $\mu_{\mathrm{O}_{2}}$ ) higher cathodic overpotentials are required to drive the oxygen chemical potential into the regime with constant vacancy concentration, and thus the limiting current regime shifts to more cathodic overpotentials in higher $p_{\mathrm{O}_{2}}$. In lower oxygen partial pressures (see Figures $3 \mathrm{c}$ and $3 \mathrm{~d}$ ) the slope $\frac{-2 e}{k T}$ regime vanishes and the cathodic current reaches a current limited regime already at very low overpotentials. This is due to the oxygen vacancy concentration being constant and equal to its equilibrium value even at very low overpotential. Since there is no effect of $p_{\mathrm{O}_{2}}$ on the oxygen vacancy concentration the current scales with $\sqrt{p_{\mathrm{O}_{2}}}$ in this regime.

Thus, in both anodic and cathodic direction we find current limited regimes. However, these current limitations are not caused by mass transport limitation, but instead are the result of the relevant defect concentrations being $\mu_{\mathrm{O}_{2}}$ independent in those regimes.

Adsorption site restriction.--So far the cathodic current scaled with $\sqrt{p_{\mathrm{O}_{2}}}$ even at very high partial pressures, since we assumed that adsorbates are sufficiently dilute, i.e. site restriction was neglected. To account for limited adsorption sites, we can extend the given model by modifying the first reaction step to include adsorption sites $\left(\mathrm{ad}_{\mathrm{a}} \mathrm{t}\right)$ for atomic oxygen (Langmuir-type dissociative adsorption). Equations 24 to 26 then transform to

$$
\begin{aligned}
& 1 / 2 \mathrm{O}_{2}+\mathrm{ad}_{(\mathrm{at})} \rightleftharpoons \mathrm{O}_{(\mathrm{ad})}
\end{aligned}
$$



$$
\begin{aligned}
& \mathrm{O}_{\mathrm{O}}^{\ddot{*}}+2 \mathrm{Fe}_{\mathrm{Fe}}^{\times} \rightleftharpoons \mathrm{O}_{\mathrm{O}}^{\times}+2 \mathrm{~h}^{\circ} \text {. }
\end{aligned}
$$

The equilibrium constant of the preceding adsorption equilibrium then becomes

$$
K_{\mathrm{ads}}=\frac{\theta_{\mathrm{at}}^{0}}{\sqrt{p_{\mathrm{O}_{2}}}\left(1-\theta_{\mathrm{at}}^{0}\right)} .
$$

From this we get

$$
\begin{gathered}
\theta_{\mathrm{at}}^{0}=\frac{K_{\mathrm{ads}} \sqrt{p_{\mathrm{O}_{2}}}}{1+K_{\mathrm{ads}} \sqrt{p_{\mathrm{O}_{2}}}}, \\
\left(1-\theta_{\mathrm{at}}^{0}\right)=\frac{1}{1+K_{\mathrm{ads}} \sqrt{p_{\mathrm{O}_{2}}}} .
\end{gathered}
$$

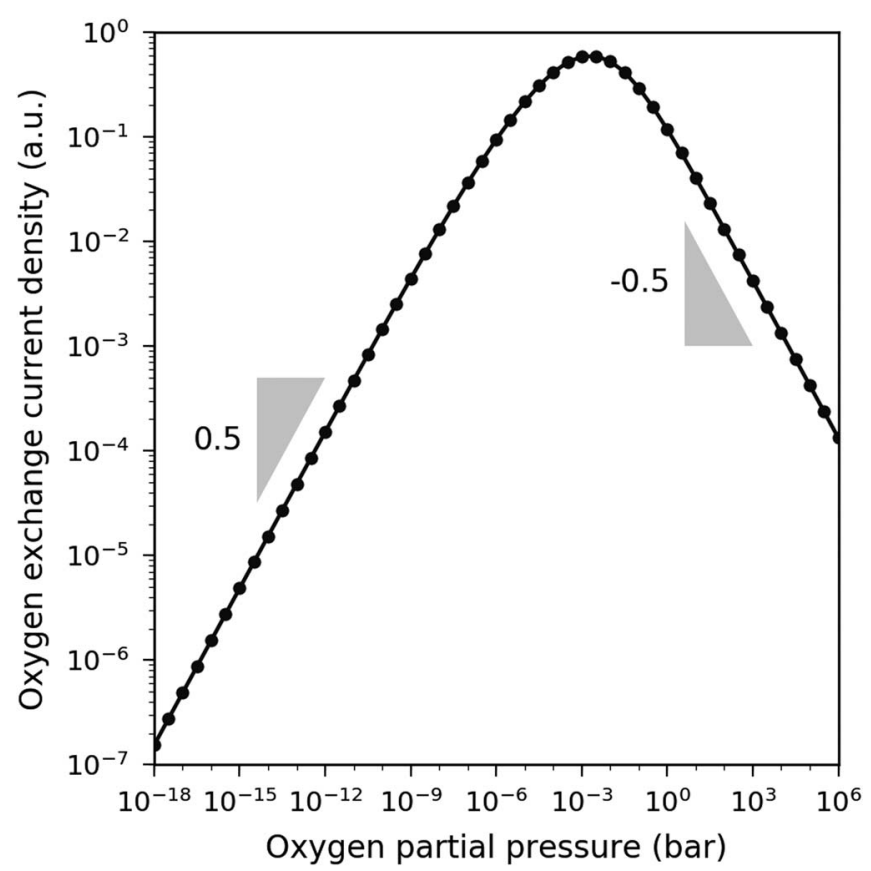

Figure 4. Oxygen exchange current density for the atomic mechanism with adsorption site restriction as a function of oxygen partial pressure, calculated by Equation 43 with $K_{\mathrm{ads}}=10 \mathrm{bar}^{-0.5}$.

Equation 34 then becomes

$j^{0}=j_{\mathrm{a}}^{0}\left(c_{\mathrm{h}}^{\mathrm{eq}}\right)^{2}\left(c_{\mathrm{Fe}}^{\mathrm{eq}}\right)^{-2} c_{\mathrm{Ox}}^{\mathrm{eq}} \frac{1}{1+K_{\mathrm{ads}} \sqrt{p_{\mathrm{O}_{2}}}}=j_{\mathrm{c}}^{0} \sqrt{p_{\mathrm{O}_{2}}} c_{\mathrm{V}}^{\mathrm{eq}} \frac{1}{1+K_{\mathrm{ads}} \sqrt{p_{\mathrm{O}_{2}}}}$.

with $j_{\mathrm{a}}^{0}=k_{\mathrm{a}} / K_{\text {ion }}$ and $j_{\mathrm{c}}^{0}=k_{\mathrm{c}} K_{\mathrm{ads}}$.

Figure 4 shows the exchange current as a function of $p_{\mathrm{O}_{2}}$ for $K_{\text {ads }}$ of $10 \mathrm{bar}^{-0.5}$, i.e. a situation where a $p_{\mathrm{O}_{2}}$ of 10 mbar leads to a surface coverage with atomic oxygen adsorbates of $50 \%$. In the low $p_{\mathrm{O}_{2}}$ regime the exchange current still increases with the square root of partial pressure; at higher $p_{\mathrm{O}_{2}}$ however, the exchange current decreases with $\sqrt{p_{\mathrm{O}_{2}}}$ due to the increasing occupation of adsorption sites.

This decrease at higher $p_{\mathrm{O}_{2}}$ is also reflected in the current-voltage curves shown in Figure 5 (calculated from Equations 33 and 43 and the Brouwer diagram in Figure 1 at $600^{\circ} \mathrm{C}$ ). At moderately high oxygen partial pressure (Figure $5 \mathrm{~b}$ ) the cathodic current still increases with $p_{\mathrm{O}_{2}}$; at around 1 bar the cathodic current reaches a maximum and then decreases with increasing $p_{\mathrm{O}_{2}}$, see Figure $5 \mathrm{a}$, in contrast to the situation without adsorption site restriction in Figure 3a. This surprising decrease of the cathodic current density with increasing $p_{\mathrm{O}_{2}}$ is due to the saturation of adsorption sites and the oxygen vacancy decrease for increasing $p_{\mathrm{O}_{2}}$. Only the limiting current for very cathodic overpotentials remains $p_{\mathrm{O}_{2}}$ independent due to the saturation of $c_{\mathrm{V}}$.

Also for anodic currents the adsorption limitation affects the current-voltage characteristics. Without site restriction, a $p_{\mathrm{O}_{2}}$ independent limiting current was found for high oxygen partial pressure (Figures $3 \mathrm{a}$ and $3 \mathrm{~b}$ ) due to the $p_{\mathrm{O}_{2}}$ independence of the hole concentration. With site restriction, however, free adsorption sites have to be available to form $\mathrm{O}_{(\mathrm{ad})}$. Those sites are strongly reduced by high $p_{\mathrm{O}_{2}}$ and thus the limiting current (which is the exchange current) decreases with increasing $p_{\mathrm{O}_{2}}$. The shape of the individual (cathodic and anodic) current-voltage curves, however, remains the same as discussed above, since the adsorption limitation only affects the exchange current density but not the overpotential dependent $c_{\mathrm{D}} / c_{\mathrm{D}}^{\text {eq }}$ defect concentration ratios in Equation 33.

\section{Current-Voltage Curves for a Molecular Mechanism}

General equations and exchange current density.-As the second mechanism we consider a molecular mechanism: A fast adsorp- 
a) Very high $p_{\mathrm{O}_{2}}$



b) High $p_{\mathrm{O}_{2}}$

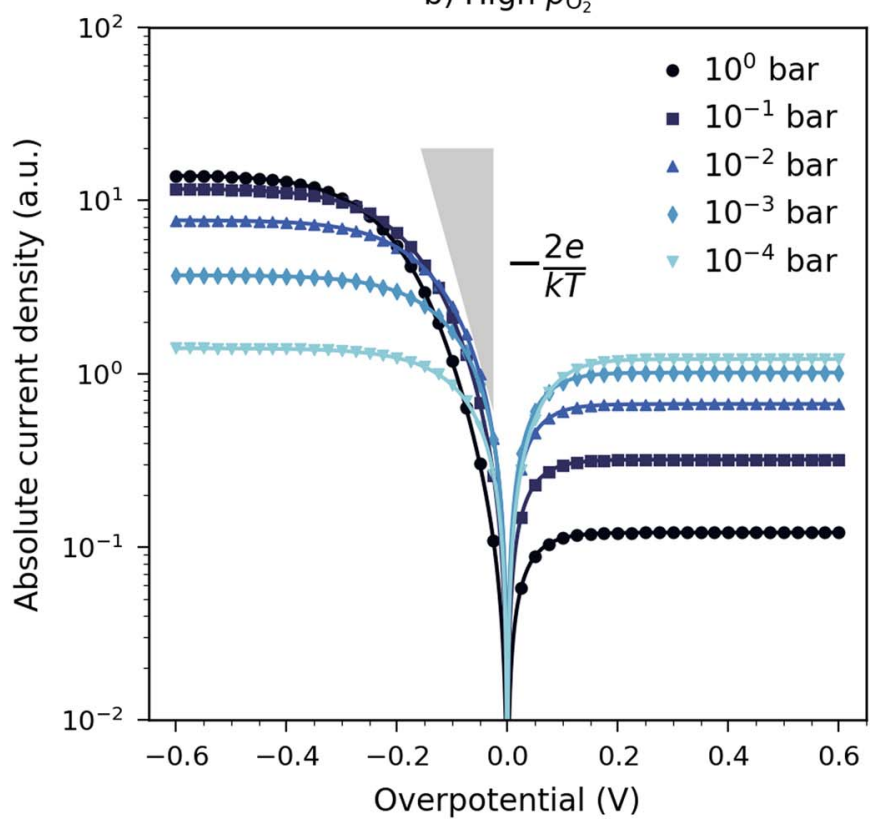

Figure 5. Current voltage characteristics for the atomic mechanism with adsorption site restriction for different oxygen partial pressures and $K_{\text {ads }}$ of 10 bar ${ }^{-0.5}$. Defect concentrations are based on the Brouwer diagram in Figure 1.

tion and partial reduction of molecular oxygen 44 is followed by rate determining dissociation with partial incorporation of the adsorbate into an oxygen vacancy 45 , and a fast incorporation of the remaining atomic oxygen dissociates with complete reduction of all incorporated O species 46.

$$
\begin{gathered}
\mathrm{O}_{2}+\mathrm{Fe}_{\mathrm{Fe}}^{\times} \rightleftharpoons \mathrm{O}_{2(\mathrm{ad})}^{-}+\mathrm{h}^{\cdot} \\
\mathrm{O}_{2(\mathrm{ad})}^{-}+\mathrm{V}_{\mathrm{O}}^{\ddot{*}} \longleftrightarrow \mathrm{O}_{\mathrm{O}}^{\cdot}+\mathrm{O}_{(\mathrm{ad})} \\
\mathrm{O}_{(\mathrm{ad})}+\mathrm{O}_{\mathrm{O}}^{\cdot}+\mathrm{V}_{\mathrm{O}}^{\ddot{*}}+3 \mathrm{Fe}_{\mathrm{Fe}}^{\times} \rightleftharpoons 2 \mathrm{O}_{\mathrm{O}}^{\times}+3 \mathrm{~h}^{\circ}
\end{gathered}
$$

The anodic and cathodic current densities are given by

$$
\begin{aligned}
& j_{\mathrm{a}}=k_{\mathrm{a}} \theta_{\mathrm{at}}^{0} c_{\mathrm{Op}} \\
& j_{\mathrm{c}}=k_{\mathrm{c}} c_{\mathrm{V}} \theta_{\text {mo }}^{-}
\end{aligned}
$$

where $\theta_{\text {at }}^{0}$ and $\theta_{\text {mo }}^{-}$are the surface coverages with neutral atomic oxygen

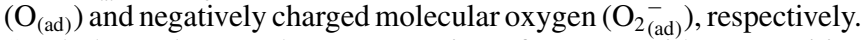
Symbol $c_{\mathrm{Op}}$ denotes the concentration of oxygen with one positive relative charge $\left(\mathrm{O}_{\mathrm{O}}^{*}\right)$. Steps 46 and 44 are assumed sufficiently fast and thus always in equilibrium with the mass action laws

$$
\begin{gathered}
K_{\text {ion }}=\frac{c_{\mathrm{Ox}}{ }^{2} c_{\mathrm{h}}{ }^{3}}{\theta_{\mathrm{at}}^{0} c_{\mathrm{Op}} c_{\mathrm{V}} c_{\mathrm{Fe}}{ }^{3}} \\
K_{\mathrm{ads}}=\frac{\theta_{\mathrm{mo}}^{-} c_{\mathrm{h}}}{p_{\mathrm{O}_{2}} c_{\mathrm{Fe}}} .
\end{gathered}
$$

Combining Equations 47 and 48 with Equations 49 and 50 gives

$$
\begin{gathered}
j_{\mathrm{a}}=j_{\mathrm{a}}^{0} c_{\mathrm{Ox}}{ }^{2} c_{\mathrm{h}}{ }^{3} c_{\mathrm{Fe}}{ }^{-3} c_{\mathrm{V}}{ }^{-1} \\
j_{\mathrm{c}}=j_{\mathrm{c}}^{0} p_{\mathrm{O}_{2}} c_{\mathrm{V}} c_{\mathrm{Fe}} c_{\mathrm{h}}{ }^{-1},
\end{gathered}
$$

with $j_{\mathrm{a}}^{0}=k_{\mathrm{a}} / K_{\text {ion }}$ and $j_{\mathrm{c}}^{0}=k_{\mathrm{c}} K_{\mathrm{ads}}$. Equation 9 thus becomes

$j=j^{0}\left[\left(\frac{c_{\mathrm{Ox}}}{c_{\mathrm{Ox}}^{\mathrm{eq}}}\right)^{2}\left(\frac{c_{\mathrm{h}}}{c_{\mathrm{h}}^{\mathrm{eq}}}\right)^{3}\left(\frac{c_{\mathrm{Fe}}}{c_{\mathrm{Fe}}^{\mathrm{eq}}}\right)^{-3}\left(\frac{c_{\mathrm{V}}}{c_{\mathrm{V}}^{\mathrm{eq}}}\right)^{-1}-\left(\frac{c_{\mathrm{V}}}{c_{\mathrm{V}}^{\mathrm{eq}}}\right)\left(\frac{c_{\mathrm{Fe}}}{c_{\mathrm{Fe}}^{\mathrm{eq}}}\right)\left(\frac{c_{\mathrm{h}}}{c_{\mathrm{h}}^{\mathrm{eq}}}\right)^{-1}\right]$,

with the partial pressure dependent exchange current density

$$
j^{0}=j_{\mathrm{a}}^{0}\left(c_{\mathrm{Ox}}^{\mathrm{eq}}\right)^{2}\left(c_{\mathrm{h}}^{\mathrm{eq}}\right)^{3}\left(c_{\mathrm{Fe}}^{\mathrm{eq}}\right)^{-3}\left(c_{\mathrm{V}}^{\mathrm{eq}}\right)^{-1}=j_{\mathrm{c}}^{0} p_{\mathrm{O}_{2}} c_{\mathrm{V}}^{\mathrm{eq}} c_{\mathrm{Fe}}^{\mathrm{eq}}\left(c_{\mathrm{h}}^{\mathrm{eq}}\right)^{-1} .
$$

In Figure 6 the exchange current density, i.e. the reaction rate of the anodic and cathodic reactions without bias, is plotted versus $p_{\mathrm{O}_{2}}$ in arbitrary units. At low oxygen partial pressure the exchange current scales with $p_{\mathrm{O}_{2}}{ }^{0.75}$. Intuitively one might assume a slope of 1 versus $p_{\mathrm{O}_{2}}$ for a molecular mechanism. However, the exchange current density depends not only on the nature of the oxygen species in the rate determining step (atomic or molecular) but also on the defect concentrations. The reason for the slope of 0.75 is that the oxygen partial pressure also acts upon the concentration of electron holes (slope of 0.25 vs. $p_{\mathrm{O}_{2}}$ ). Since holes enter the cathodic reaction rate with a

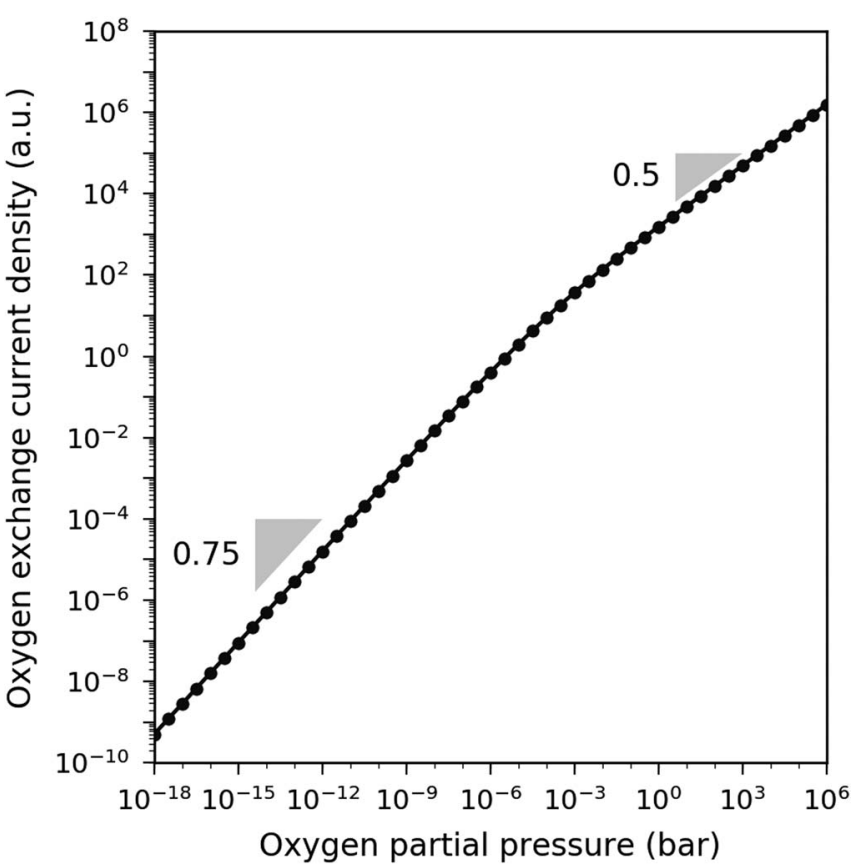

Figure 6. Oxygen exchange current density for the molecular mechanism as a function of oxygen partial pressure, calculated by Equation 54. 
negative exponent, it partly counteracts the direct $p_{\mathrm{O}_{2}}$ effect via adsorbates, resulting in a $p_{\mathrm{O}_{2}}$ dependency of 0.75 . Alternatively, one may consider the anodic direction in Equation 54, where the oxygen partial pressure only affects the exchange current density indirectly by changing the electron hole concentration (slope 0.25 ). This also results in a slope of 0.75 since three holes are involved in the anodic reaction rate. Oxygen vacancies are irrelevant as their concentration is constant in the low $p_{\mathrm{O}_{2}}$ regime.

At high oxygen partial pressure the exchange current scales only with $\sqrt{p_{\mathrm{O}_{2}}}$, and this might be interpreted in terms of an atomic mechanism. However, this is again due to $p_{\mathrm{O}_{2}}$ induced changes in defect concentrations. In the cathodic direction the reaction order of one with respect to $p_{\mathrm{O}_{2}}$ (direct effect via adsorbates) is partly counter-balanced by the oxygen vacancy concentration decreasing with $\sqrt{p_{\mathrm{O}_{2}}}$ and thus a reaction order of 0.5 results. Alternatively, we may again consider the anodic direction, where the indirect effect (oxygen vacancy concentration decreasing with increasing $p_{\mathrm{O}_{2}}$ ) also leads to a slope of 0.5 since the anodic exchange current density is proportional to $\left(c_{\mathrm{V}}^{\mathrm{eq}}\right)^{-1}$. The reaction orders with respect to electron holes play no role here, as the electron hole concentration is constant in high oxygen partial pressures.

This example shows that the common connection of molecular mechanisms with a linear scaling of the exchange current density with $p_{\mathrm{O}_{2}}$ (slope of 1 ) is not mandatory. Rather, the $p_{\mathrm{O}_{2}}$ dependence of $j^{0}$ is a complex interplay between the nature of the oxygen adsorbate in the rate determining step, the involved defects and their $p_{\mathrm{O}_{2}}$ dependence. In this example, the oxygen exchange current density at high $p_{\mathrm{O}_{2}}$ scales with $p_{\mathrm{O}_{2}}{ }^{0.5}$, which is often interpreted as a sign of an atomic reaction mechanism.

From Equations 53 and 54 we can calculate the partial pressure dependent current-voltage characteristics, which are shown in Figure 7. Below we discuss how these curves can be interpreted as a result of the LSF defect chemistry.

Anodic polarization.-In high oxygen partial pressures (see Figures $7 \mathrm{a}$ and $7 \mathrm{~b}$ ) the current-voltage curves are exponential and thus show straight lines in the Tafel plot. Since the overpotential acts by driving defect concentrations away from their equilibrium values, the reason for the shape of these curves is a consequence of the Brouwer diagram. Oxygen vacancies and electron holes both affect the anodic reaction rate (and thus current density) by the preceding equilibrium in Reaction 44. However, the electron hole concentration is virtually constant at these high oxygen chemical potentials. Thus, the effect of anodic overpotential is to decrease the concentration of oxygen vacancies, which causes an increase of the net current density, as the anodic reaction order with respect to vacancies is -1 . The slope of $\ln j_{\mathrm{a}}$ vs. $\eta$ is $\frac{2 e}{k T}$ and this value consist of three factors:

$$
\frac{\mathrm{d} \ln j_{\mathrm{a}}}{\mathrm{d} \eta}=\frac{\mathrm{d} \ln j_{\mathrm{a}}}{\mathrm{d} \ln c_{\mathrm{V}}} \frac{\mathrm{d} \ln c_{\mathrm{V}}}{\mathrm{d} \mu_{\mathrm{O}_{2}}} \frac{\mathrm{d} \mu_{\mathrm{O}_{2}}}{\mathrm{~d} \eta}=-1 \frac{-1}{2 k T} 4 e=\frac{2 e}{k T} .
$$

The first factor $\frac{\mathrm{d} \ln j_{\mathrm{a}}}{\mathrm{d} \ln c_{\mathrm{V}}}$ is the reaction order with respect to oxygen vacancies, i.e. -1 for this specific mechanism, the second one $\frac{d \ln c_{V}}{d \mu_{O}}$ is a consequence of the Brouwer diagram and the third one comes from Nernst's Equation 12. The current also scales with $p_{\mathrm{O}_{2}}{ }^{0.5}$ which reflects the slope of the exchange current (see Figure 6); the reason for this accelerating effect of $p_{\mathrm{O}_{2}}$ is only an indirect one due to the oxygen vacancy concentration scaling with $p_{\mathrm{O}_{2}}{ }^{-0.5}$.

In lower oxygen partial pressures (see Figures $7 \mathrm{c}$ and $7 \mathrm{~d}$ ) the current-voltage curves are also exponential, but the slope varies from $\frac{3 e}{k T}$ at lower overpotentials to $\frac{2 e}{k T}$ at higher overpotentials; the overpotential of this transition depends on $p_{\mathrm{O}_{2}}$. Again, the slopes can be understood as a consequence of the LSF Brouwer diagram. Oxygen vacancies and electrons enter the anodic rate equation, but in the low $\mu_{\mathrm{O}_{2}}$ regime (i.e. for low overpotential and low $p_{\mathrm{O}_{2}}$ ) the concentration of vacancies is constant and the overpotential acts by increasing the electron hole concentration. The slope of $\ln j_{\mathrm{a}}$ vs. $\eta\left(\frac{3 e}{k T}\right)$ is again the sum of three terms:

$$
\frac{\mathrm{d} \ln j_{\mathrm{a}}}{\mathrm{d} \eta}=\frac{\mathrm{d} \ln j_{\mathrm{a}}}{\mathrm{d} \ln c_{\mathrm{h}}} \frac{\mathrm{d} \ln c_{\mathrm{h}}}{\mathrm{d} \mu_{\mathrm{O}_{2}}} \frac{\mathrm{d} \mu_{\mathrm{O}_{2}}}{\mathrm{~d} \eta}=3 \frac{1}{4 k T} 4 e=\frac{3 e}{k T} .
$$

At higher overpotentials (and thus high $\mu_{\mathrm{O}_{2}}$ ) the electron hole concentration reaches a plateau and the oxygen vacancy concentration starts to decrease, resulting in a slope of $\frac{2 e}{k T}$ as described above. This also explains why the overpotential of the transition from $\frac{3 e}{k T}$ to $\frac{2 e}{k T}$ depends on $p_{\mathrm{O}_{2}}$, since at low oxygen partial pressures higher overpotentials are required to reach the oxygen chemical potential where the minority charge carrier changes from electron holes to oxygen vacancies. At low overpotential the current scales with $p_{\mathrm{O}_{2}}{ }^{0.75}$ which is due to the hole concentration increasing with $p_{\mathrm{O}_{2}}{ }^{0.25}$ in accordance with the slope of the exchange current density. At higher overpotentials the current scales only with $p_{\mathrm{O}_{2}}{ }^{0.5}$, since there the vacancies are the $\mu_{\mathrm{O}_{2}}$ dependent defects.

Cathodic polarization.-Under mildly cathodic polarization and in very high oxygen partial pressures (Figures $7 \mathrm{a}$ and $7 \mathrm{~b}$ ), the oxygen incorporation rate increases exponentially with overpotential with an exponent of $-\frac{2 e}{k T}$ and the $p_{\mathrm{O}_{2}}$ dependence of $j_{\mathrm{c}}$ in this regime is $j_{\mathrm{c}} \propto p_{\mathrm{O}_{2}}{ }^{0.5}$. At higher overpotentials the curves bend toward a slope of $-\frac{e}{k T}$, the overpotentials where this transition occurs depends again on $p_{\mathrm{O}_{2}}$. Furthermore, $j_{\mathrm{c}}$ scales with $p_{\mathrm{O}_{2}}{ }^{0.75}$. This behavior can again be explained as a direct consequence of the LSF defect chemistry. Both oxygen vacancies and electron holes enter the cathodic current density in Equation 53. At low polarization (and thus in the high $\mu_{\mathrm{O}_{2}}$ regime) the electron hole concentration is essentially constant, but the oxygen vacancy concentration changes with $\mu_{\mathrm{O}_{2}}$. An applied overpotential thus acts by increasing the oxygen vacancy concentration and the resulting slope of $-\frac{2 e}{k T}$ consists of three factors:

$$
\frac{\mathrm{d} \ln j_{\mathrm{c}}}{d \eta}=\frac{\mathrm{d} \ln j_{\mathrm{c}}}{\mathrm{d} \ln c_{\mathrm{V}}} \frac{\mathrm{d} \ln c_{\mathrm{V}}}{\mathrm{d} \mu_{\mathrm{O}_{2}}} \frac{\mathrm{d} \mu_{\mathrm{O}_{2}}}{\mathrm{~d} \eta}=1 \frac{-1}{2 k T} 4 e=-\frac{2 e}{k T} .
$$

The current scales with $p_{\mathrm{O}_{2}}{ }^{0.5}$ in this regime, since the reaction order due to molecular gas adsorbates $\left(\nu_{\mathrm{p}, \mathrm{c}}=1\right)$ is partly compensated by the oxygen vacancy concentration which decreases with $p_{\mathrm{O}_{2}}{ }^{0.5}$.

At sufficiently large cathodic overpotentials the oxygen vacancy concentration reaches a plateau and the electron holes start to decrease with increasingly cathodic overpotential. The overpotential thus acts now by decreasing the electron hole concentration resulting is a slope of $\frac{e}{k T}$ according to

$$
\frac{d \ln j_{\mathrm{c}}}{d \eta}=\frac{d \ln j_{\mathrm{c}}}{d \ln c_{\mathrm{h}}} \frac{d \ln c_{\mathrm{h}}}{d \mu_{\mathrm{O}_{2}}} \frac{d \mu_{\mathrm{O}_{2}}}{d \eta}=-1 \frac{1}{4 k T} 4 e=-\frac{e}{k T} .
$$

The overpotential at which this transition from $-\frac{2 e}{k T}$ to $-\frac{e}{k T}$ occurs depends on $p_{\mathrm{O}_{2}}$ since high oxygen partial pressures require more cathodic overpotentials to reach the $\mu_{\mathrm{O}_{2}}$ regime where electron holes begin to change with $\mu_{\mathrm{O}_{2}}$. The partial pressure dependence of the current-density in the $-\frac{e}{k T}$ regime is $p_{\mathrm{O}_{2}}{ }^{0.75}$, since here the reaction order due to molecular gas adsorbates $\left(\nu_{\mathrm{p}, \mathrm{c}}\right)$ of 1 gets reduced by 0.25 due to the electron hole concentration increasing with $p_{\mathrm{O}_{2}}{ }^{0.25}$. In lower oxygen partial pressures (Figures $7 \mathrm{c}$ and $7 \mathrm{~d}$ ) the slope is constant at $-\frac{e}{k T}$ since even at very low cathodic polarization the oxygen chemical potential is already in the regime where the oxygen vacancy concentration is constant. Consequently, the only effect of the overpotential on the current occurs via decreasing the electron hole concentration.

In contrast to the atomic mechanism (Figure 3) neither anodic nor cathodic limiting currents are found for the molecular mechanisms considered here (Figure 7). This is due to the fact that anodic as well as cathodic currents depend on both, oxygen vacancies and holes, see Equation 33. In accordance with the Brouwer diagram either $c_{\mathrm{V}}$ or $c_{\mathrm{h}}$ has to depend on $\mu_{\mathrm{O}_{2}}$ and thus exponential current-voltage curves are found in all cases.

Adsorption site limitation.-In the previous section (atomic mechanism) we discussed the effect of adsorption site limitation for the case 
a) Very high $p_{\mathrm{O}_{2}}$



c) Medium $p_{\mathrm{O}_{2}}$

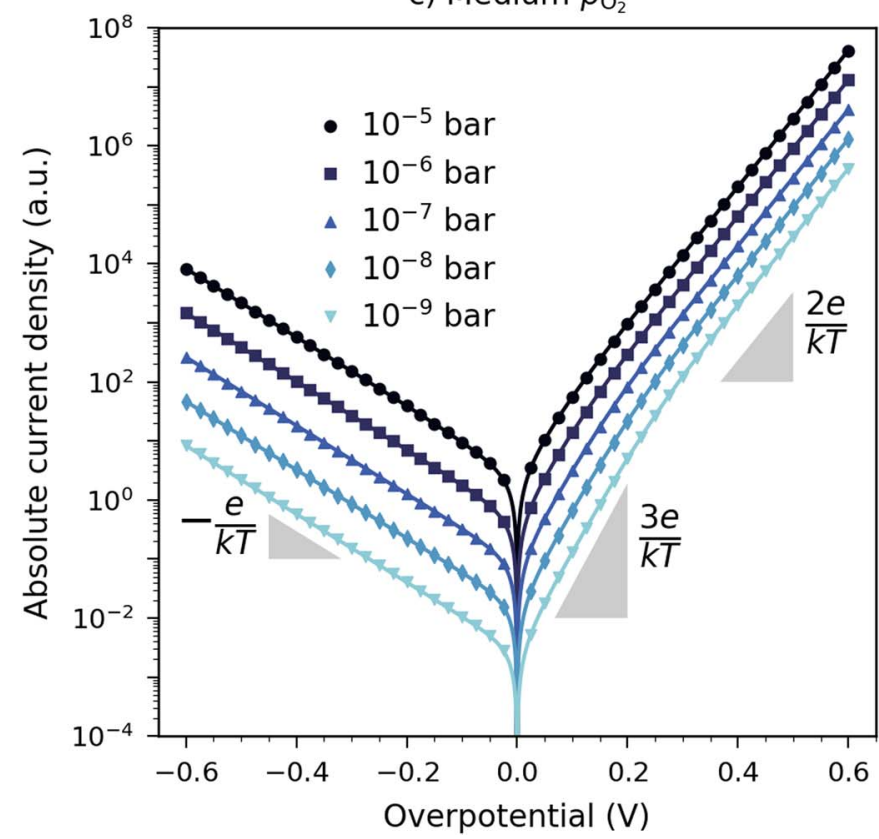

b) High $p_{\mathrm{O}_{2}}$

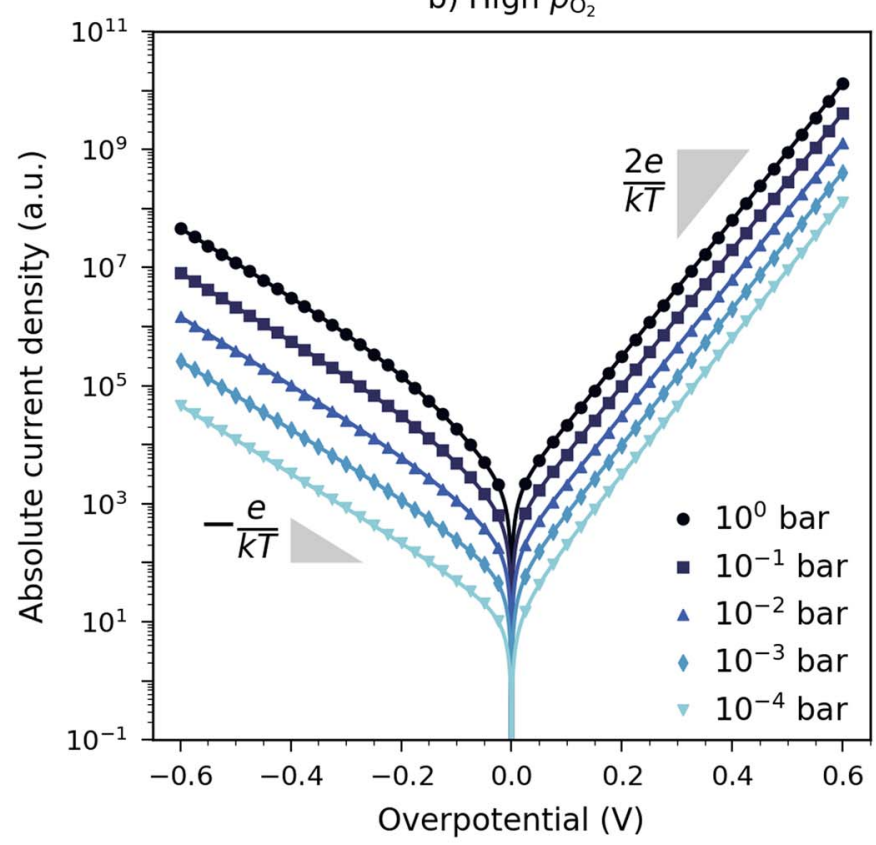

d) Low $p_{\mathrm{O}_{2}}$

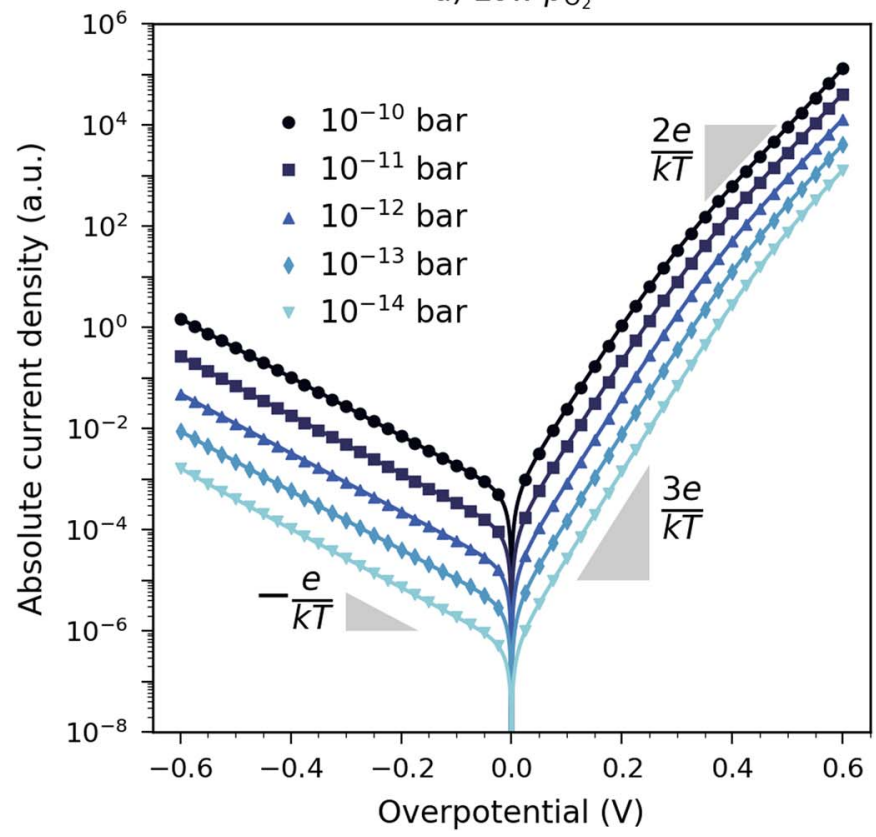

Figure 7. Current voltage characteristics for the molecular mechanism, calculated by Equation 53, for different oxygen partial pressures. Defect concentrations are based on the Brouwer diagram in Figure 1.

of neutral adsorption, i.e. without charge transfer prior to the rate determining step. There, we found that the adsorption site only causes a decreasing exchange current-density above a certain $p_{\mathrm{O}_{2}}$ threshold, while leaving the individual current-voltage relations unchanged. Below, we discuss the effects of site restriction on the current-voltage and current-partial pressure relations for our molecular mechanism, i.e. for the case where there is an electron transfer prior to the rate limiting step.

We again assume a Langmuir-type adsorption, i.e. we neglect any variation of the adsorption enthalpy with coverage and specifically neglect any surface potential changes. For the sake of simplicity, we neglect site limitation for the intermediate atomic adsorbates $\left(\mathrm{O}_{(\mathrm{ad})}\right)$, and further assume different adsorption sites for molecular and atomic adsorbates. Equations 44 to 46 then transform to

$$
\begin{aligned}
& \mathrm{O}_{2}+\mathrm{ad}_{(\mathrm{mo})}+\mathrm{Fe}_{\mathrm{Fe}}^{\times}+\rightleftharpoons \mathrm{O}_{2(\mathrm{ad})}^{-}+\mathrm{h}^{\cdot} \\
& \mathrm{O}_{2(\mathrm{ad})}^{-}+\mathrm{V}_{\mathrm{O}}^{\ddot{ }} \longleftrightarrow \mathrm{O}_{\mathrm{O}}^{\cdot}+\mathrm{O}_{(\mathrm{ad})}+\mathrm{ad}_{(\mathrm{mo})} \\
& \mathrm{O}_{(\mathrm{ad})}+\mathrm{O}_{\mathrm{O}}^{\cdot}+\mathrm{V}_{\mathrm{O}}^{\ddot{ }}+3 \mathrm{Fe}_{\mathrm{Fe}}^{\times} \rightleftharpoons 2 \mathrm{O}_{\mathrm{O}}^{\times}+3 \mathrm{~h}^{*}
\end{aligned}
$$

The equilibrium constant of the preceding adsorption equilibrium then becomes

$$
K_{\mathrm{ads}}=\frac{\theta_{\mathrm{mo}}^{-} c_{\mathrm{h}}}{p_{\mathrm{O}_{2}}\left(1-\theta_{\mathrm{mo}}^{-}\right) c_{\mathrm{Fe}}} .
$$




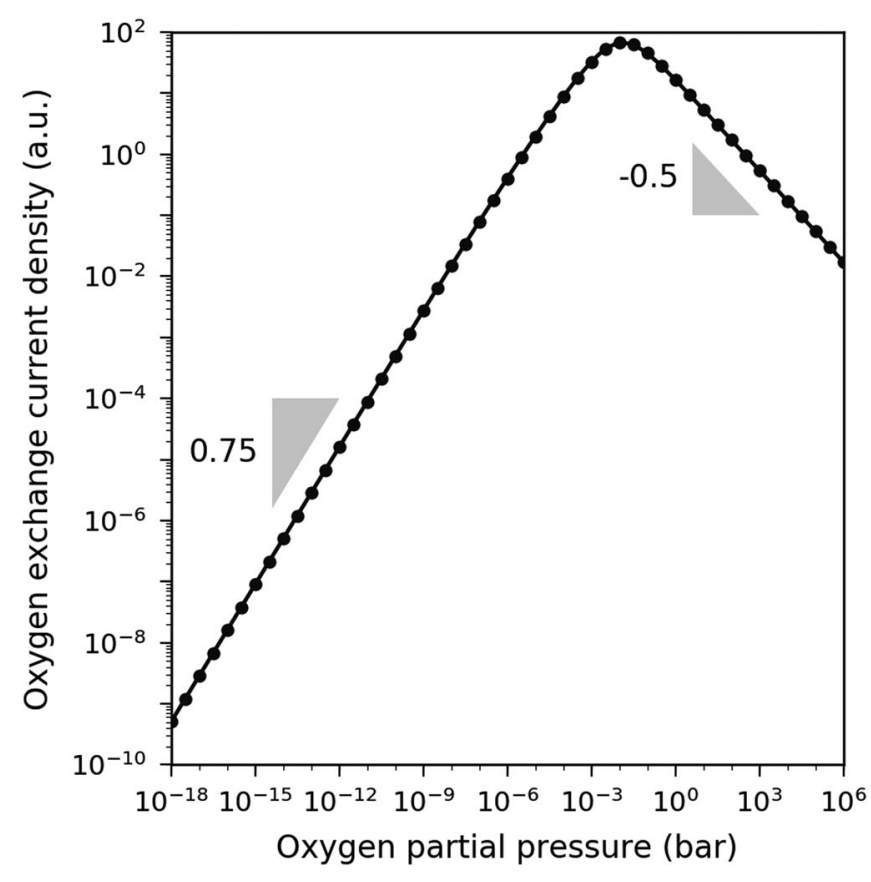

Figure 8. Oxygen exchange current density for the molecular mechanism with adsorption site restriction as a function of oxygen partial pressure, calculated by Equation 65 for $K_{\text {ads }}=59.4 \mathrm{bar}^{-1}$.

From this we get

$$
\begin{gathered}
\theta_{\mathrm{mo}}^{-}=\frac{1}{1+K_{\mathrm{ads}} p_{\mathrm{O}_{2}} c_{\mathrm{Fe}} / c_{\mathrm{h}}} K_{\mathrm{ads}} p_{\mathrm{O}_{2}} \frac{c_{\mathrm{Fe}}}{c_{\mathrm{h}}}, \\
\left(1-\theta_{\mathrm{mo}}^{-}\right)=\frac{1}{1+K_{\mathrm{ads}} p_{\mathrm{O}_{2}} c_{\mathrm{Fe}} / c_{\mathrm{h}}} .
\end{gathered}
$$

Equation 54 then becomes

$$
\begin{aligned}
j^{0} & =j_{\mathrm{a}}^{0}\left(c_{\mathrm{h}}^{\mathrm{eq}}\right)^{3}\left(c_{\mathrm{V}}^{\mathrm{eq}}\right)^{-1}\left(c_{\mathrm{Fe}}^{\mathrm{eq}}\right)^{-3}\left(c_{\mathrm{Ox}}^{\mathrm{eq}}\right)^{2} \frac{1}{1+K_{\mathrm{ads}} p_{\mathrm{O}_{2}} c_{\mathrm{Fe}}^{\mathrm{eq}} / c_{\mathrm{h}}^{\mathrm{eq}}} \\
& =j_{\mathrm{c}}^{0} p_{\mathrm{O}_{2}}\left(c_{\mathrm{V}}^{\mathrm{eq}}\right)\left(c_{\mathrm{h}}^{\mathrm{eq}}\right)^{-1}\left(c_{\mathrm{Fe}}^{\mathrm{eq}}\right) \frac{1}{1+K_{\mathrm{ads}} p_{\mathrm{O}_{2}} c_{\mathrm{Fe}}^{\mathrm{eq}} / c_{\mathrm{h}}^{\mathrm{eq}}}
\end{aligned}
$$

with $j_{\mathrm{a}}^{0}=k_{\mathrm{a}} / K_{\text {ion }}$ and $j_{\mathrm{c}}^{0}=k_{\mathrm{c}} K_{\mathrm{ads}}$.

We assume an equilibrium constant for the adsorption $\left(K_{\mathrm{ads}}\right)$ of $59.4 \mathrm{bar}^{-1}$ such that the surface coverage $\left(\theta_{\mathrm{mo}}^{-}\right)$is $50 \%$ at an oxygen partial pressure of 10 mbar; similar to the atomic mechanism discussed in Adsorption site restriction section. The additional factor of 0.594 compared to Adsorption site restriction section is due to the involvement of electron holes in the adsorption equilibrium. Figure 8 shows the exchange current density as a function of $p_{\mathrm{O}_{2}}$. In the low $p_{\mathrm{O}_{2}}$ regime $\left(p_{\mathrm{O}_{2}}<<1 / K_{\text {ads }}\right)$ the site restriction is irrelevant and the exchange current still increases with ${p_{\mathrm{O}_{2}}}^{0.75}$ as described above. At higher $p_{\mathrm{O}_{2}}$ however, the exchange current decreases with $p_{\mathrm{O}_{2}}{ }^{-0.5}$ due to the increasing occupation of adsorption sites. In principle, electron holes are involved in the adsorption equilibrium, and thus their ( $p_{\mathrm{O}_{2}}$ dependent) concentration also affects the adsorbate concentration. However, for this specific value of $K_{\text {ads }}$ the hole concentration is almost constant in the regime where the site restriction becomes relevant. Thus, in the high $p_{\mathrm{O}_{2}}$ regime the oxygen partial pressure acts only by decreasing oxygen vacancy concentration and thus the exchange current-density decreases with $p_{\mathrm{O}_{2}}{ }^{0.5}$. Equivalently, we may consider the anodic $j^{0}$ in Equation 43 and see that higher $p_{\mathrm{O}_{2}}$ decreases the number of free adsorption sites needed to form $\mathrm{O}_{2(\mathrm{ad})}^{-}$.
For the current under polarization, Equation 53 has to be replaced by

$$
\begin{aligned}
j= & j^{0} \frac{1+K_{\mathrm{ads}} p_{\mathrm{O}_{2}} c_{\mathrm{Fe}}^{\mathrm{eq}} / c_{\mathrm{h}}^{\mathrm{eq}}}{1+K_{\mathrm{ads}} p_{\mathrm{O}_{2}} c_{\mathrm{Fe}} / c_{\mathrm{h}}}\left[\left(\frac{c_{\mathrm{Ox}}}{c_{\mathrm{Ox}}^{\mathrm{eq}}}\right)^{2}\left(\frac{c_{\mathrm{h}}}{c_{\mathrm{h}}^{\mathrm{eq}}}\right)^{3}\left(\frac{c_{\mathrm{Fe}}}{c_{\mathrm{Fe}}^{\mathrm{eq}}}\right)^{-3}\left(\frac{c_{\mathrm{V}}}{c_{\mathrm{V}}^{\mathrm{eq}}}\right)^{-1}\right. \\
& \left.-\left(\frac{c_{\mathrm{V}}}{c_{\mathrm{V}}^{\mathrm{eq}}}\right)\left(\frac{c_{\mathrm{Fe}}}{c_{\mathrm{Fe}}^{\mathrm{eq}}}\right)\left(\frac{c_{\mathrm{h}}}{c_{\mathrm{h}}^{\mathrm{eq}}}\right)^{-1}\right] .
\end{aligned}
$$

Figure 9 shows the corresponding current-voltage curves for a wide $p_{\mathrm{O}_{2}}$ range. At low oxygen partial pressures (Figures $9 \mathrm{c}$ and $9 \mathrm{~d}$ ) the current-voltage curves are very similar to the ones without adsorption site restriction discussed above (Figures $7 \mathrm{c}$ and $7 \mathrm{~d}$ ) in both anodic and cathodic direction. However, at higher oxygen partial pressures (Figures 9a and 9b) the situation is very different since the adsorption site restriction becomes relevant and depends on the overpotential.

In the anodic direction, the current reaches a maximum at an oxygen partial pressure of 100 mbar and scales with $p_{\mathrm{O}_{2}}{ }^{-0.5}$ at higher oxygen partial pressures, which reflects the $p_{\mathrm{O}_{2}}$ dependence of the exchange current density. The involvement of electron holes in the adsorption equilibrium plays no role here, since at high $p_{\mathrm{O}_{2}}$, where site restriction is relevant, the electron hole concentration does not change with $p_{\mathrm{O}_{2}}$ or anodic overpotential. Therefore, the accelerating effect of $p_{\mathrm{O}_{2}}$ (due to $c_{\mathrm{V}} \propto p_{\mathrm{O}_{2}}{ }^{-0.5}$ ) gets overcompensated by a decelerating effect due to the number of free adsorption sites decreasing linearly with increasing $p_{\mathrm{O}_{2}}$. The shape of the individual current-voltage curves is unaffected by the adsorption site restriction.

In the cathodic direction, the relation between current density, overpotential and partial pressure is more complex. This is because electron holes are involved in the adsorption equilibrium (Equation 59) and the surface coverage is thus tied to the defect chemistry. Accordingly, site occupancy is affected by the overpotential. More specific, high cathodic overpotentials lower the hole concentration and thus increase the coverage with $\mathrm{O}_{2(\text { ad) }}^{-}$, possibly approaching full occupancy. At moderate oxygen partial pressures $\left(10^{-5}\right.$ to $10^{-4}$ bar $)$ the current first increases exponentially with cathodic overpotential; the corresponding Tafel slope is $-\frac{e}{k T}$. As described above, this slope originates from the decreasing electron hole concentration. Since the surface coverage has not reached saturation, the current also increases with $p_{\mathrm{O}_{2}}$. At more cathodic overpotentials, however, the surface coverage reaches saturation due to the low electron hole concentration. The overpotential thus neither affects the oxygen vacancy concentration nor the surface coverage and thus the current becomes constant, cf. Equation 48. Furthermore, since the surface is saturated with adsorbates the current also becomes $p_{\mathrm{O}_{2}}$ independent.

At even higher oxygen partial pressures, the current first increases with a Tafel slope of $-\frac{2 e}{k T}$ before reaching a current limited regime. Here, the surface is saturated with adsorbates already at zero overpotential and thus an applied overpotential only acts by increasing the oxygen vacancy concentration, which leads to the Tafel slope of $-\frac{2 e}{k T}$. At higher cathodic overpotentials, the oxygen vacancy concentration reaches a plateau and the current becomes constant.

This example mechanism shows that, even without considering any surface potential changes, the adsorbate concentration is tied to the overpotential due to the involvement of defects in the adsorption equilibrium. In the simple case without adsorption site restriction this causes exponential current-voltage curves with a Tafel slope of $-\frac{e}{k T}$ in the low $\mu_{\mathrm{O}_{2}}$ regime. If the finite number of adsorption sites is considered, this dependency of the surface coverage on the overpotential (via defect concentrations) leads to a situation, where the surface becomes saturated with adsorbates even at moderately low $p_{\mathrm{O}_{2}}$ if the overpotential is sufficiently cathodic. Moreover, this saturation can cause limiting currents also for the mechanism under investigation, in contrast to the case without saturation (Figure 7). 
a) Very high $p_{\mathrm{O}_{2}}$



c) Medium $p_{\mathrm{O}_{2}}$

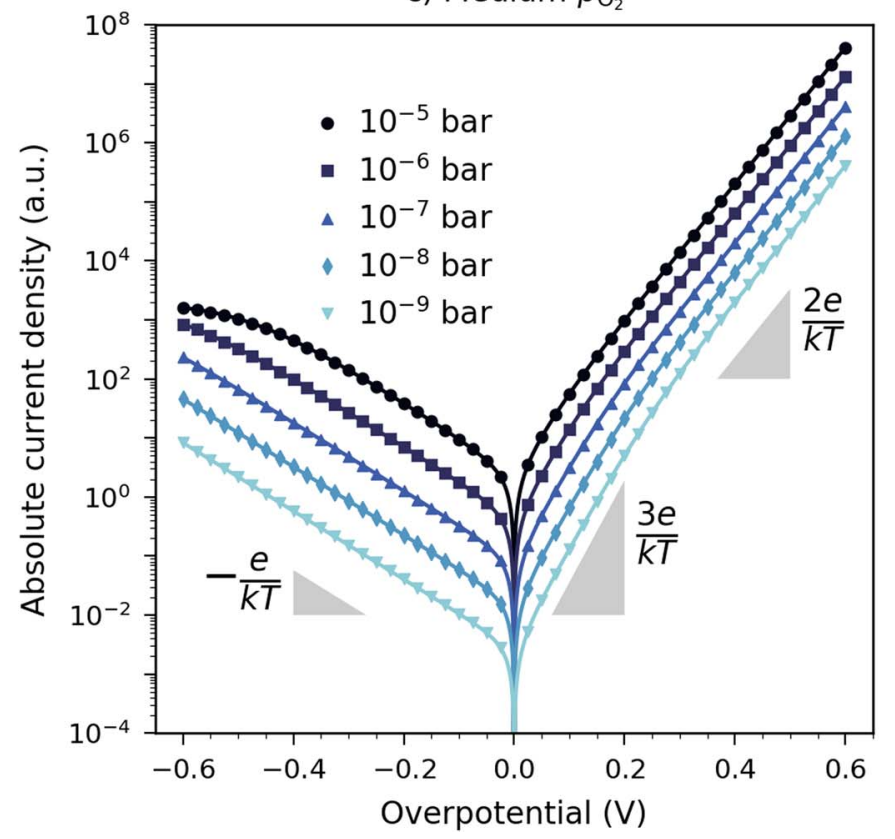

b) High $p_{\mathrm{O}_{2}}$

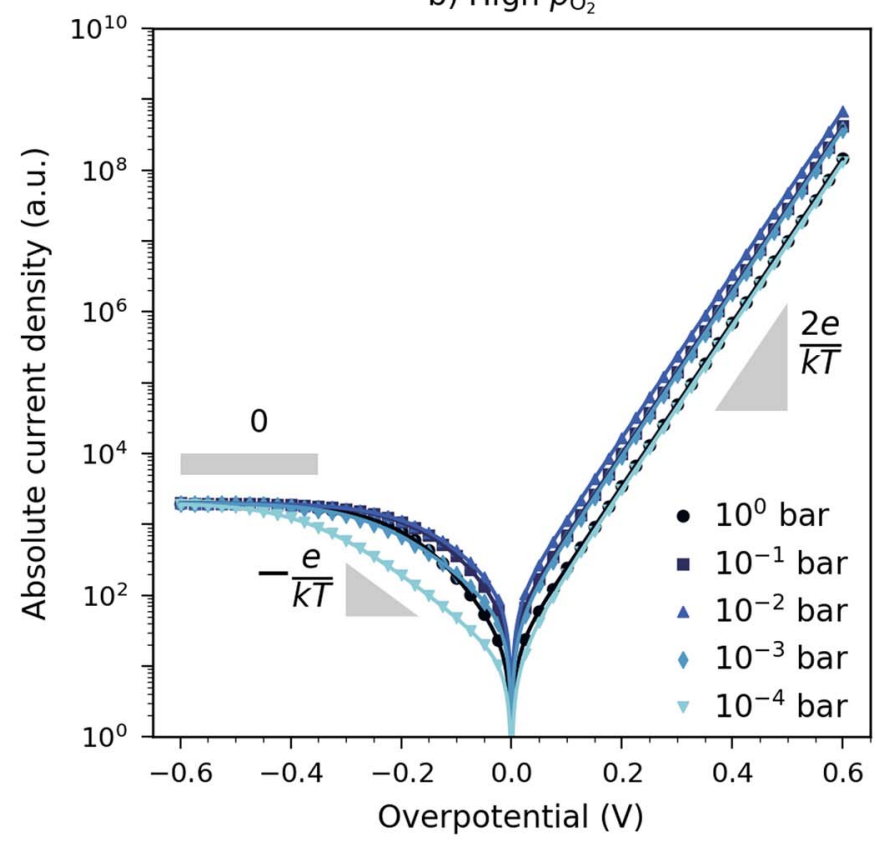

d) Low $p_{\mathrm{O}_{2}}$

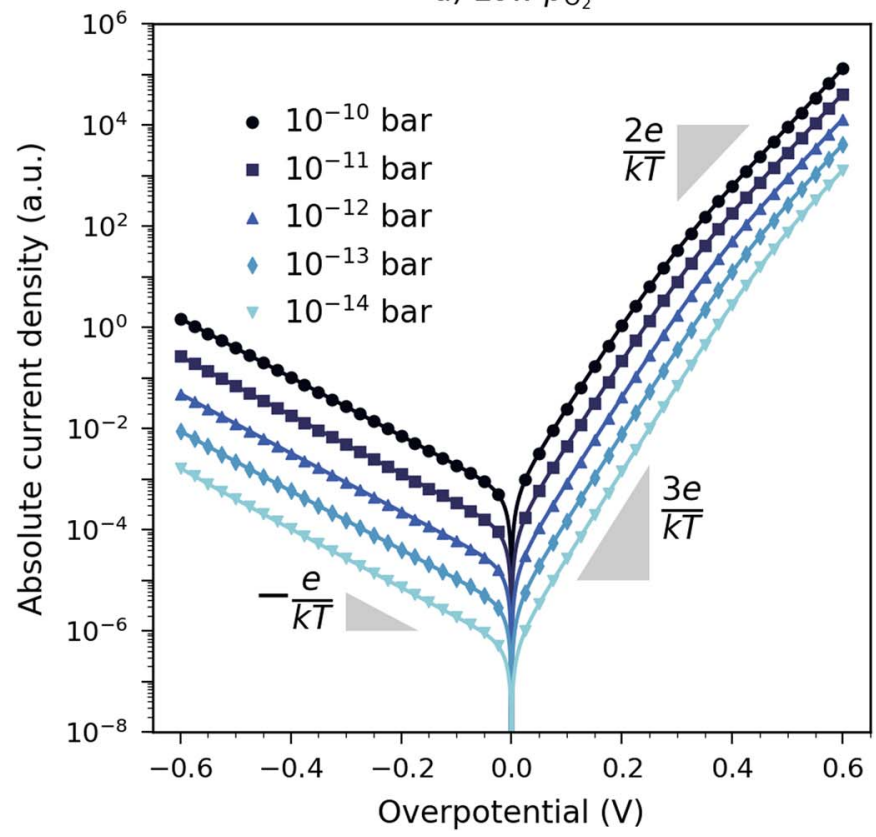

Figure 9. Current voltage characteristics for the molecular mechanism with adsorption site restriction for different oxygen partial pressures. Defect concentrations are based on the Brouwer diagram in Figure 1.

\section{Generalized Tafel-Slope and Partial Pressure Dependence}

In the discussion of the atomic and molecular mechanism in the previous sections we derived the dependencies of the current densities on oxygen partial pressure and the overpotential as a consequence of defect concentrations, based on the known LSF Brouwer diagram. In the following, we derive generalized expressions for the slopes of the anodic and cathodic current densities with respect to $p_{\mathrm{O}_{2}}$ and overpotential, i.e. for the exponents of the $p_{\mathrm{O}_{2}}$ power laws and the Tafel slopes.

The anodic and cathodic current densities in equilibrium (Equations 4 and 5) have to be in accordance with the mass action laws of the defect chemical reactions, i.e. the oxygen exchange (Equation 22) and the iron disproportionation (Equation 23). For the sake of simplicity we neglect $\mathrm{O}_{\mathrm{O}}^{\times}$and $\mathrm{Fe}_{\mathrm{Fe}}^{\times}$as defect species with changing concentrations and thus only oxygen vacancies $\mathrm{V}_{\mathrm{O}}$, electrons $\mathrm{e}^{\prime}$ and electron holes $h^{*}$ are left from the defect model of LSF (or other acceptor doped mixed conductors). For those we can specify rate Equations 2 and 3, still assuming $\Delta \chi=0$ :

$$
\begin{aligned}
& j_{\mathrm{a}}=j_{\mathrm{a}}^{0} c_{\mathrm{V}}{ }^{{ }_{\mathrm{V}, \mathrm{a}}} c_{\mathrm{h}}{ }^{{ }_{\mathrm{h}, \mathrm{a}}} c_{\mathrm{e}}{ }^{{ }_{\mathrm{e}, \mathrm{a}}} p_{\mathrm{O}_{2}}{ }^{{ }_{\mathrm{p}, \mathrm{a}}} \\
& j_{\mathrm{c}}=j_{\mathrm{c}}^{0} c_{\mathrm{V}}{ }^{{ }{ }_{\mathrm{V}, \mathrm{c}}} c_{\mathrm{h}}{ }^{{ }_{\mathrm{h}, \mathrm{c}}} c_{\mathrm{e}}{ }^{{ }_{\mathrm{e}, \mathrm{c}}} p_{\mathrm{O}_{2}}{ }^{{ }_{\mathrm{p}, \mathrm{c}}} .
\end{aligned}
$$

Equilibrium means $j_{\mathrm{a}}=j_{\mathrm{c}}$ and thus

$\frac{j_{\mathrm{c}}^{0}}{j_{\mathrm{a}}^{0}}=K_{\mathrm{kin}}=c_{\mathrm{V}}{ }^{\left(v_{\mathrm{V}, \mathrm{a}}-v_{\mathrm{V}, \mathrm{c}}\right)} c_{\mathrm{h}}{ }^{\left(\nu_{\mathrm{h}, \mathrm{a}}-v_{\mathrm{h}, \mathrm{c}}\right)} c_{\mathrm{e}}{ }^{\left(v_{\mathrm{e}, \mathrm{a}}-v_{\mathrm{e}, \mathrm{c}}\right)} p_{\mathrm{O}_{2}}{ }^{\left(v_{\mathrm{p}, \mathrm{a}}-v_{\mathrm{p}, \mathrm{c}}\right)}$. 
Moreover, we know from Equation 23 that

$$
K_{\mathrm{i}} c_{\mathrm{Fe}}^{2}=K_{\mathrm{i}}^{\prime}=c_{\mathrm{h}} c_{\mathrm{e}}
$$

and therefore $c_{\mathrm{e}}=K_{\mathrm{i}}^{\prime} / c_{\mathrm{h}}$. Hence,

$$
K_{\mathrm{kin}} / K_{\mathrm{i}}^{\left(v_{\mathrm{e}, \mathrm{a}}-v_{\mathrm{e}, \mathrm{c}}\right)}=c_{\mathrm{V}}{ }^{\left(\nu_{\mathrm{V}, \mathrm{a}}-v_{\mathrm{V}, \mathrm{c}}\right)} c_{\mathrm{h}}{ }^{\left(\left(\nu_{\mathrm{h}, \mathrm{a}}-v_{\mathrm{h}, \mathrm{c}}\right)-\left(v_{\mathrm{e}, \mathrm{a}}-v_{\mathrm{e}, \mathrm{c}}\right)\right)} p_{\mathrm{O}_{2}}{ }^{\left(v_{\mathrm{p}, \mathrm{a}}-v_{\mathrm{p}, \mathrm{c}}\right)} \text {. }
$$

This has to be in accordance with the mass action law for the oxygen incorporation. With $K_{\mathrm{Ox}}^{\prime}=K_{\mathrm{Ox}}{ }^{2} \frac{c_{\mathrm{Fe}}{ }^{4}}{c_{\mathrm{Ox}}}$ and constant $c_{\mathrm{Fe}}$ and $c_{\mathrm{Ox}}$ we can write

$$
\left(K_{\mathrm{ox}}^{\prime}\right)^{n}=\left(\frac{c_{\mathrm{h}}{ }^{4}}{p_{\mathrm{O}_{2}} c_{\mathrm{V}}^{2}}\right)^{n} .
$$

Actually, in the mass action law $n$ can still be chosen arbitrarily, and in Equation $22 n=0.5$ was used (with still variable concentrations $c_{\mathrm{Ox}}$ and $c_{\mathrm{Fe}}$ ). However, $n$ becomes well defined by the equivalence of Equations 71 and 72 , which requires

$$
\begin{gathered}
\nu_{\mathrm{V}, \mathrm{a}}-\nu_{\mathrm{V}, \mathrm{c}}=-2 n \\
\left(\nu_{\mathrm{h}, \mathrm{a}}-\nu_{\mathrm{e}, \mathrm{a}}\right)-\left(\nu_{\mathrm{h}, \mathrm{c}}-\nu_{\mathrm{e}, \mathrm{c}}\right)=4 n \\
\nu_{\mathrm{p}, \mathrm{a}}-\nu_{\mathrm{p}, \mathrm{c}}=-n .
\end{gathered}
$$

For simple mechanisms, we have $\nu_{\mathrm{p}, \mathrm{a}}=0$ and then $n$ is simply the cathodic reaction order with respect to the oxygen partial pressure $\nu_{\mathrm{p}, \mathrm{c}}$, i.e. 0.5 for atomic or 1 for molecular mechanisms. Furthermore, from Equations 72 and 70 we get

$$
\begin{gathered}
\frac{\mathrm{d} \ln K_{\mathrm{ox}}^{\prime}}{\mathrm{d} \mu_{\mathrm{O}_{2}}}=4 \frac{\mathrm{d} \ln c_{\mathrm{h}}}{\mathrm{d} \mu_{\mathrm{O}_{2}}}-2 \frac{\mathrm{d} \ln c_{\mathrm{V}}}{\mathrm{d} \mu_{\mathrm{O}_{2}}}-\frac{\mathrm{d} \ln p_{\mathrm{O}_{2}}}{\mathrm{~d} \mu_{\mathrm{O}_{2}}}=0 \\
\frac{\mathrm{d} \ln K_{\mathrm{i}}^{\prime}}{\mathrm{d} \mu_{\mathrm{O}_{2}}}=\frac{\mathrm{d} \ln c_{\mathrm{h}}}{\mathrm{d} \mu_{\mathrm{O}_{2}}}+\frac{\mathrm{d} \ln c_{\mathrm{e}}}{\mathrm{d} \mu_{\mathrm{O}_{2}}}=0 .
\end{gathered}
$$

Equations 76 and 77 imply

$$
\begin{gathered}
\frac{\mathrm{d} \ln c_{\mathrm{V}}}{\mathrm{d} \mu_{\mathrm{O}_{2}}}=2 \frac{\mathrm{d} \ln c_{\mathrm{h}}}{\mathrm{d} \mu_{\mathrm{O}_{2}}}-\frac{1}{2 k T} \\
\frac{\mathrm{d} \ln c_{\mathrm{e}}}{\mathrm{d} \mu_{\mathrm{O}_{2}}}=-\frac{\mathrm{d} \ln c_{\mathrm{h}}}{\mathrm{d} \mu_{\mathrm{O}_{2}}} .
\end{gathered}
$$

Partial pressure dependence.-From Equations 67 and 68 we can deduce slopes according to

$$
\begin{aligned}
\frac{\mathrm{d} \ln j_{\mathrm{a}}}{\mathrm{d} \ln p_{\mathrm{O}_{2}}} & =\frac{\mathrm{d} \ln j_{\mathrm{a}}}{\mathrm{d} \mu_{\mathrm{O}_{2}}} \frac{\mathrm{d} \mu_{\mathrm{O}_{2}}}{\mathrm{~d} \ln p_{\mathrm{O}_{2}}} \\
& =k T\left(\nu_{\mathrm{V}, \mathrm{a}} \frac{\mathrm{d} \ln c_{\mathrm{V}}}{\mathrm{d} \mu_{\mathrm{O}_{2}}}+\nu_{\mathrm{h}, \mathrm{a}} \frac{\mathrm{d} \ln c_{\mathrm{h}}}{\mathrm{d} \mu_{\mathrm{O}_{2}}}+\nu_{\mathrm{e}, \mathrm{a}} \frac{\mathrm{d} \ln c_{\mathrm{e}}}{\mathrm{d} \mu_{\mathrm{O}_{2}}}+v_{\mathrm{p}, \mathrm{a}} \frac{1}{k T}\right) \\
\frac{\mathrm{d} \ln j_{\mathrm{c}}}{\mathrm{d} \ln p_{\mathrm{O}_{2}}} & =\frac{\mathrm{d} \ln j_{\mathrm{c}}}{\mathrm{d} \mu_{\mathrm{O}_{2}}} \frac{\mathrm{d} \mu_{\mathrm{O}_{2}}}{\mathrm{~d} \ln p_{\mathrm{O}_{2}}} \\
& =k T\left(\nu_{\mathrm{V}, \mathrm{c}} \frac{\mathrm{d} \ln c_{\mathrm{V}}}{\mathrm{d} \mu_{\mathrm{O}_{2}}}+v_{\mathrm{h}, \mathrm{c}} \frac{\mathrm{d} \ln c_{\mathrm{h}}}{\mathrm{d} \mu_{\mathrm{O}_{2}}}+v_{\mathrm{e}, \mathrm{c}} \frac{\mathrm{d} \ln c_{\mathrm{e}}}{\mathrm{d} \mu_{\mathrm{O}_{2}}}+v_{\mathrm{p}, \mathrm{c}} \frac{1}{k T}\right) .
\end{aligned}
$$

Combining Equations 80 and 81 with Equations 78 and 79 gives

$$
\begin{aligned}
& \frac{\mathrm{d} \ln j_{\mathrm{a}}}{\operatorname{dln} p_{\mathrm{O}_{2}}}=k T\left(v_{\mathrm{h}, \mathrm{a}}-v_{\mathrm{e}, \mathrm{a}}+2 v_{\mathrm{V}, \mathrm{a}}\right) \frac{\mathrm{d} \ln c_{\mathrm{h}}}{\mathrm{d} \mu_{\mathrm{O}_{2}}}-\frac{1}{2} v_{\mathrm{V}, \mathrm{a}}+v_{\mathrm{p}, \mathrm{a}}=q_{\mathrm{a}} \\
& \frac{\mathrm{d} \ln j_{\mathrm{c}}}{\operatorname{dln} p_{\mathrm{O}_{2}}}=k T\left(\nu_{\mathrm{h}, \mathrm{c}}-v_{\mathrm{e}, \mathrm{c}}+2 v_{\mathrm{V}, \mathrm{c}}\right) \frac{\mathrm{d} \ln c_{\mathrm{h}}}{\mathrm{d} \mu_{\mathrm{O}_{2}}}-\frac{1}{2} \nu_{\mathrm{V}, \mathrm{c}}+v_{\mathrm{p}, \mathrm{c}}=q_{\mathrm{c}} .
\end{aligned}
$$

Symbols $q_{\mathrm{a}}$ and $q_{\mathrm{c}}$ denote the empirical power law exponents with respect to $p_{\mathrm{O}_{2}}$. Including the relations 73,74 and 75 into these considerations shows that $q_{\mathrm{a}}=q_{\mathrm{c}}$. Thus, for a given overpotential the partial pressure dependence is the same for the anodic and cathodic reaction, it is only a function of the oxygen partial pressure itself. Consequently,

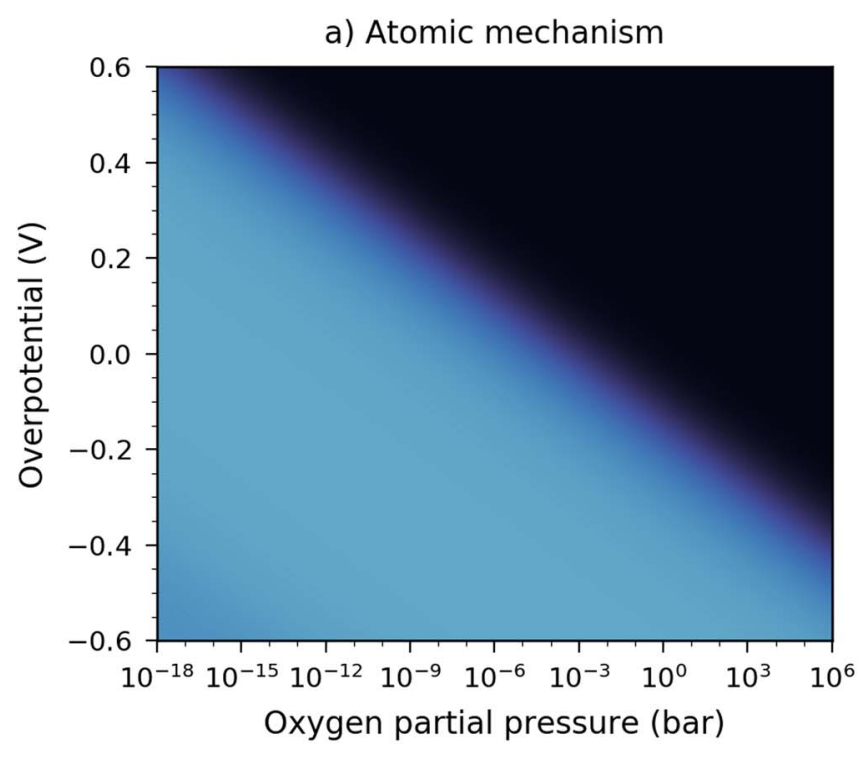

b) Molecular mechanism
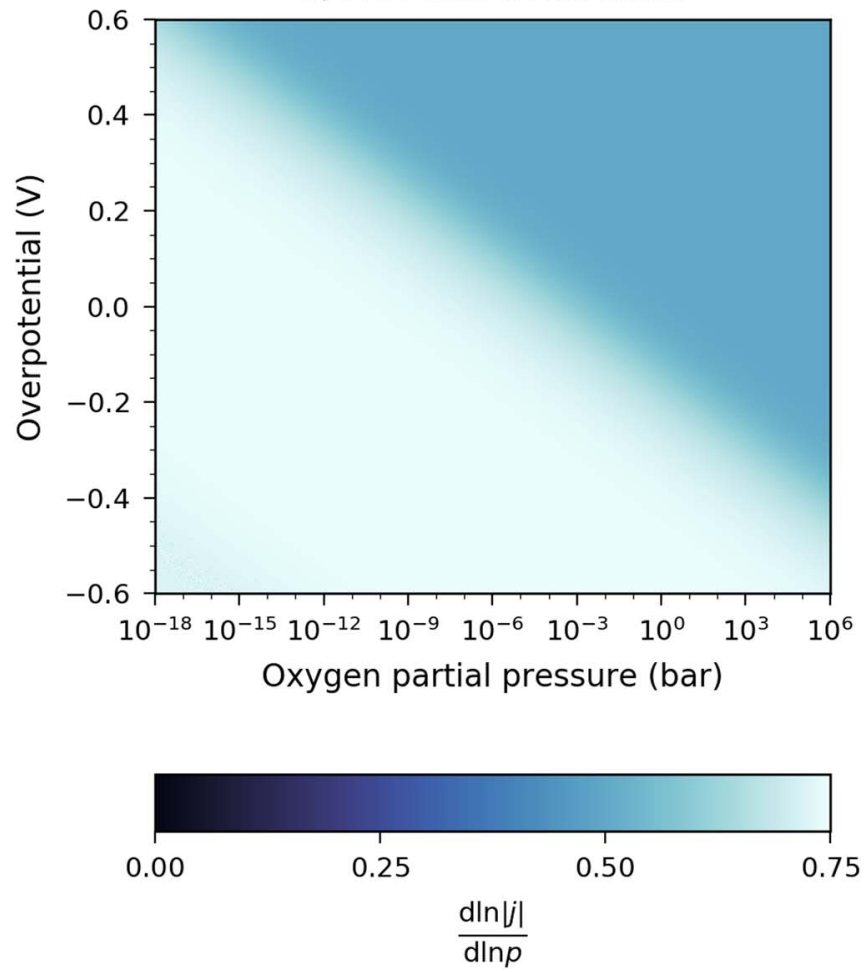

Figure 10. Partial pressure dependency of the current-density for the atomic mechanism (a) and the molecular mechanism (b) as a function of partial pressure and overpotential.

this is also the partial pressure dependence of the net current density at this overpotential and the $p_{\mathrm{O}_{2}}$ dependence of the exchange current density for equilibrium. (Please note: Despite their identical $p_{\mathrm{O}_{2}}$ dependences for a given overpotential, $j_{\mathrm{a}}$ and $j_{\mathrm{c}}$ usually differ strongly in their absolute value, unless close to equilibrium. Hence, mostly only one of the two determines the net current density.) In Figures 10a and $10 \mathrm{~b}$ the exponents of the $p_{\mathrm{O}_{2}}$ dependence of anodic and cathodic currents are shown as a function of $p_{\mathrm{O}_{2}}$ and $\eta$ for the atomic and molecular mechanism without site restriction.

Actually, the slope $\frac{\mathrm{d} \ln j}{\operatorname{dn} p_{\mathrm{O}_{2}}}$ only depends on $\mu_{\mathrm{O}_{2}}$, which is also visible when comparing these $p_{\mathrm{O}_{2}}$ dependencies with the defect concentrations displayed in Figure 11. Two defect regimes can be distinguished: 
a) Oxygen vacancies

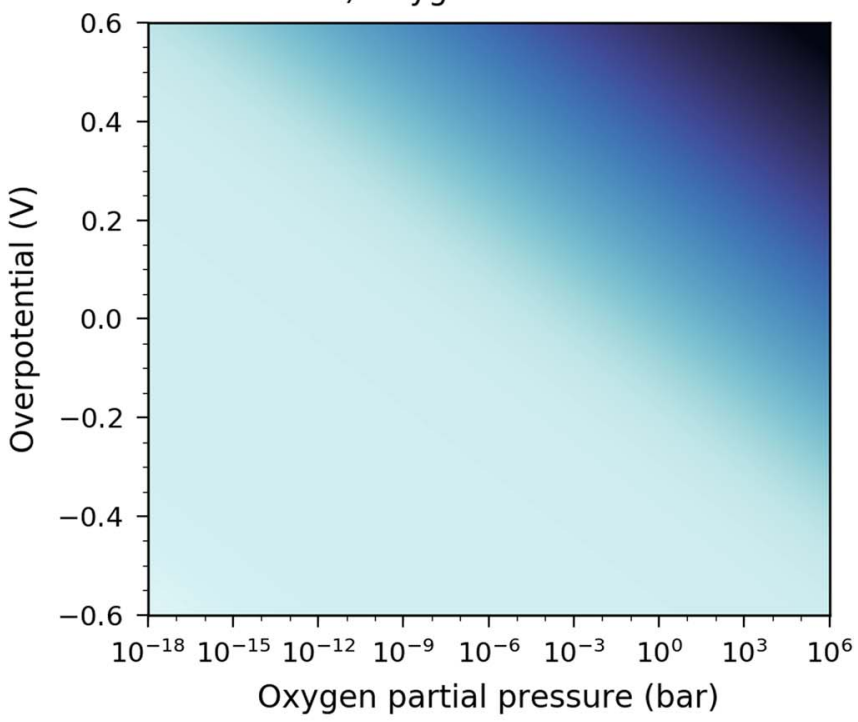

b) Electron holes

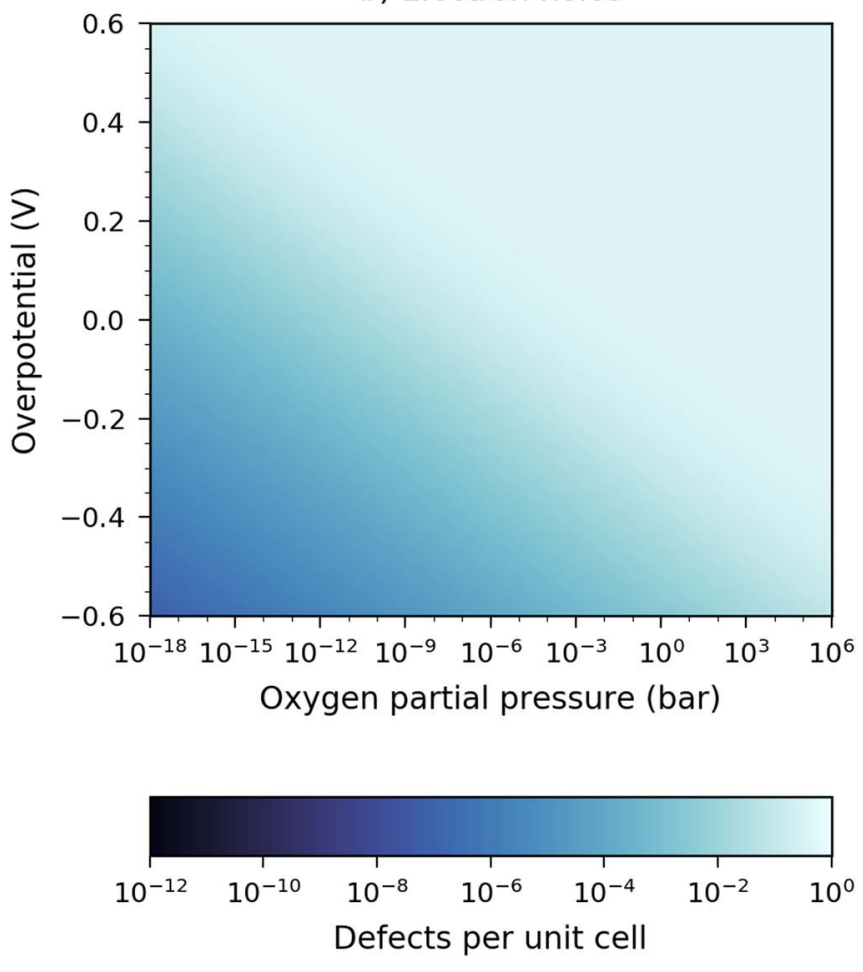

Figure 11. Extended LSF Brouwer diagram: Oxygen vacancy (a) and electron hole (b) concentrations as a function of partial pressure and overpotential.

At low $p_{\mathrm{O}_{2}}$ and negative overpotential (resulting in low $\mu_{\mathrm{O}_{2}}$ ) electron holes are the minority charge carrier and thus the $p_{\mathrm{O}_{2}}$ dependencies are determined by the electron hole reaction orders of the specific mechanisms. At high $p_{\mathrm{O}_{2}}$ and positive overpotentials (high $\mu_{\mathrm{O}_{2}}$ ) oxygen vacancies are the minority and consequently the $p_{\mathrm{O}_{2}}$ dependence of the current density depends on the reaction orders of oxygen vacancies. The equivalence of the $p_{\mathrm{O}_{2}}$ dependence of cathodic and anodic currents also means that any measured difference in the $p_{\mathrm{O}_{2}}$ dependence for anodic and cathodic voltages is simply caused by different regimes in the Brouwer diagram (i.e. different $p_{\mathrm{O}_{2}}$ ). In other words, $p_{\mathrm{O}_{2}}$ dependencies of $j$ change, when the current-voltage curves cross the color front line in Figure 11.
Tafel slopes.-From Equations 67 and 68 we get

$$
\begin{aligned}
\frac{\mathrm{d} \ln j_{\mathrm{a}}}{\mathrm{d} \eta} & =\nu_{\mathrm{V}, \mathrm{a}} \frac{\mathrm{d} \ln c_{\mathrm{V}}}{\mathrm{d} \eta}+v_{\mathrm{h}, \mathrm{a}} \frac{\mathrm{d} \ln c_{\mathrm{h}}}{\mathrm{d} \eta}+\nu_{\mathrm{e}, \mathrm{a}} \frac{\mathrm{d} \ln c_{\mathrm{e}}}{\mathrm{d} \eta}+v_{\mathrm{p}, \mathrm{a}} \frac{\mathrm{d} \ln p_{\mathrm{O}_{2}}}{\mathrm{~d} \eta} \\
& =\frac{\mathrm{d} \mu_{\mathrm{O}_{2}}}{\mathrm{~d} \eta}\left(\nu_{\mathrm{V}, \mathrm{a}} \frac{\mathrm{d} \ln c_{\mathrm{V}}}{\mathrm{d} \mu_{\mathrm{O}_{2}}}+\nu_{\mathrm{h}, \mathrm{a}} \frac{\mathrm{d} \ln c_{\mathrm{h}}}{\mathrm{d} \mu_{\mathrm{O}_{2}}}+v_{\mathrm{e}, \mathrm{a}} \frac{\mathrm{d} \ln c_{\mathrm{e}}}{\mathrm{d} \mu_{\mathrm{O}_{2}}}\right) \\
& =4 e\left(\nu_{\mathrm{V}, \mathrm{a}} \frac{\mathrm{d} \ln c_{\mathrm{V}}}{\mathrm{d} \mu_{\mathrm{O}_{2}}}+\nu_{\mathrm{h}, \mathrm{a}} \frac{\mathrm{d} \ln c_{\mathrm{h}}}{\mathrm{d} \mu_{\mathrm{O}_{2}}}+\nu_{\mathrm{e}, \mathrm{a}} \frac{\mathrm{d} \ln c_{\mathrm{e}}}{\mathrm{d} \mu_{\mathrm{O}_{2}}}\right)
\end{aligned}
$$

and

$$
\begin{aligned}
\frac{\mathrm{d} \ln j_{\mathrm{c}}}{\mathrm{d} \eta} & =\nu_{\mathrm{V}, \mathrm{c}} \frac{\mathrm{d} \ln c_{\mathrm{V}}}{\mathrm{d} \eta}+\nu_{\mathrm{h}, \mathrm{c}} \frac{\mathrm{d} \ln c_{\mathrm{h}}}{\mathrm{d} \eta}+\nu_{\mathrm{e}, \mathrm{c}} \frac{\mathrm{d} \ln c_{\mathrm{e}}}{\mathrm{d} \eta}+\nu_{\mathrm{p}, \mathrm{c}} \frac{\mathrm{d} \ln p_{\mathrm{O}_{2}}}{\mathrm{~d} \eta} \\
& =\frac{\mathrm{d} \mu_{\mathrm{O}_{2}}}{\mathrm{~d} \eta}\left(\nu_{\mathrm{V}, \mathrm{c}} \frac{\mathrm{d} \ln c_{\mathrm{V}}}{\mathrm{d} \mu_{\mathrm{O}_{2}}}+\nu_{\mathrm{h}, \mathrm{c}} \frac{\mathrm{d} \ln c_{\mathrm{h}}}{\mathrm{d} \mu_{\mathrm{O}_{2}}}+\nu_{\mathrm{e}, \mathrm{c}} \frac{\mathrm{d} \ln c_{\mathrm{e}}}{\mathrm{d} \mu_{\mathrm{O}_{2}}}\right) \\
& =4 e\left(\nu_{\mathrm{V}, \mathrm{c}} \frac{\mathrm{d} \ln c_{\mathrm{V}}}{\mathrm{d} \mu_{\mathrm{O}_{2}}}+\nu_{\mathrm{h}, \mathrm{c}} \frac{\mathrm{d} \ln c_{\mathrm{h}}}{\mathrm{d} \mu_{\mathrm{O}_{2}}}+\nu_{\mathrm{e}, \mathrm{c}} \frac{\mathrm{d} \ln c_{\mathrm{e}}}{\mathrm{d} \mu_{\mathrm{O}_{2}}}\right) .
\end{aligned}
$$

By combining Equations 84 and 85 with Equations 78 and 79 we get

$$
\begin{aligned}
& \frac{\mathrm{d} \ln j_{\mathrm{a}}}{\mathrm{d} \eta}=4 e \frac{\mathrm{d} \ln c_{\mathrm{h}}}{\mathrm{d} \mu_{\mathrm{O}_{2}}}\left(v_{\mathrm{h}, \mathrm{a}}-v_{\mathrm{e}, \mathrm{a}}+2 \nu_{\mathrm{V}, \mathrm{a}}\right)-\frac{2 e}{k T} v_{\mathrm{V}, \mathrm{a}} \\
& \frac{\mathrm{d} \ln j_{\mathrm{c}}}{\mathrm{d} \eta}=4 e \frac{\mathrm{d} \ln c_{\mathrm{h}}}{\mathrm{d} \mu_{\mathrm{O}_{2}}}\left(\nu_{\mathrm{h}, \mathrm{c}}-v_{\mathrm{e}, \mathrm{c}}+2 \nu_{\mathrm{V}, \mathrm{c}}\right)-\frac{2 e}{k T} v_{\mathrm{V}, \mathrm{c}} .
\end{aligned}
$$

From Equations 87, 74, 73 and 75 we thus obtain

$$
\frac{\mathrm{d} \ln j_{\mathrm{c}}}{\mathrm{d} \eta}=4 e \frac{\mathrm{d} \ln c_{\mathrm{h}}}{\mathrm{d} \mu_{\mathrm{O}_{2}}}\left(\nu_{\mathrm{h}, \mathrm{a}}-v_{\mathrm{e}, \mathrm{a}}+2 \nu_{\mathrm{V}, \mathrm{a}}\right)-\frac{2 e}{k T}\left(\nu_{\mathrm{V}, \mathrm{a}}+2 n\right) .
$$

Accordingly, for a given overpotential the Tafel-slopes of the anodic and cathodic currents differ by $\frac{4 e n}{k T}$. This simply reflects the fact that for a changing overpotential the anodic and cathodic currents change in a different manner. Therefore, one of them always dominates sufficiently far from equilibrium. In each direction, however, the slope is still only a function of $\mu_{\mathrm{O}_{2}}$, irrespective of the parameter changing $\mu_{\mathrm{O}_{2}}\left(p_{\mathrm{O}_{2}}\right.$ or $\left.\eta\right)$. An equivalent expression for the cathodic oxygen reduction current on Pr-doped ceria was derived by Chueh et al. in Ref. 46. The generalized expressions 86 and 88 also show that the splitting of Tafel slopes in three factors performed in the specific discussion (e.g. Equation 55) is already a simplification. Those factors are still present in the generalized expressions but are complemented by additional terms.

Figures $12 \mathrm{a}$ and $12 \mathrm{~b}$ display the Tafel slopes of the two mechanisms discussed in this work sections as a function of partial pressure and overpotential. Comparing these to the LSF defect concentrations shown in Figure 11 reveals two facts: First, two regimes can be distinguished, and these follow the minority charge carrier regimes in Figure 11. At low $p_{\mathrm{O}_{2}}$ and negative overpotential (low $\mu_{\mathrm{O}_{2}}$ ) the Tafel slopes are determined by the reaction order of electron holes. At high $\mu_{\mathrm{O}_{2}}$, oxygen vacancies are in minority and the Tafel slopes depend on the reaction order of oxygen vacancies. Second, the Tafel slopes of the anodic and cathodic currents differ by $\frac{2 e}{k T}$ and $\frac{4 e}{k T}$ respectively, over the entire $p_{\mathrm{O}_{2}}$ and $\eta$ range, as long as we stay in one defect regime. This also implies that knowledge of the nature of the oxygen species in the rate determining step (atomic or molecular) can be obtained from the anodic and cathodic Tafel slopes, provided both are still in the same minority charge carrier regime. A difference of $\frac{4 e}{k T}$, for example, results from an anodic slope of $\frac{3 e}{k T}$ and a cathodic slope of $-\frac{1 e}{k T}$ and indicates a molecular species in the rate determining step. Hence, such a slope analysis of the current-voltage curves can be a simple but powerful approach for obtaining mechanistic information already without further detailed analysis of reaction models. However, one has to keep in mind that it works only if the defect regime does not change and thus, for example, not in Figure 7b.

Oxygen exchange reaction resistance.-Lastly, we briefly discuss the oxygen exchange resistance predicted by this model. The area 

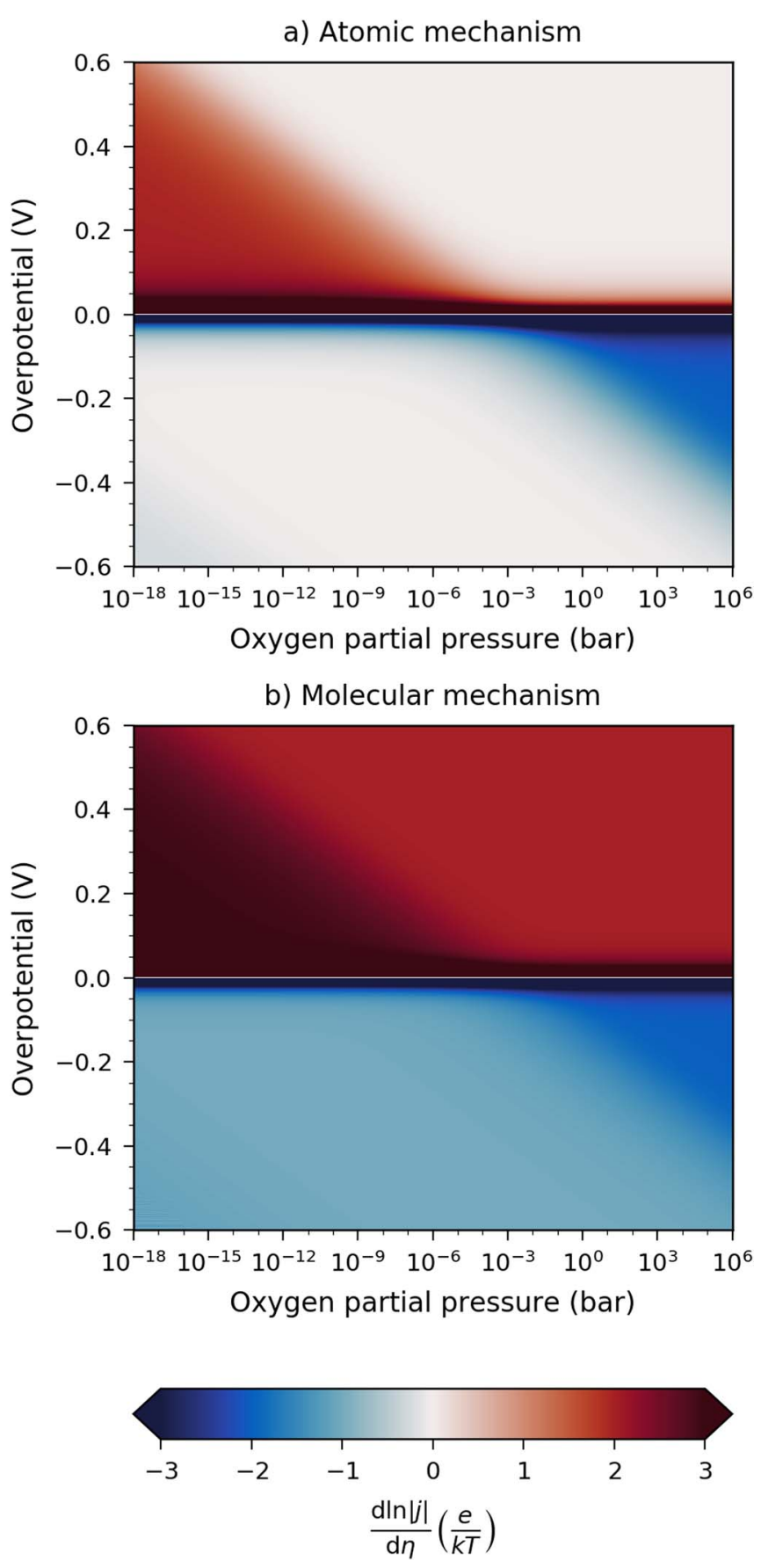

Figure 12. Tafel slopes for the atomic mechanism (a) and the molecular mechanism (b) as a function of partial pressure and overpotential.

specific reaction resistance is given by the inverse derivative of the current density with respect to the overpotential:

$$
R=\frac{\mathrm{d} \eta}{\mathrm{d} j}=\left(\frac{\mathrm{d} j}{\mathrm{~d} \eta}\right)^{-1}
$$

For the sake of simplicity, we limit ourselves to the simple case of equilibrium conditions, i.e. at $\eta=0$. Then, Equation 89 leads to

$$
R^{\mathrm{eq}}=\frac{k T}{4 e n j^{0}} .
$$

The detailed derivation of this expression is given in appendix A. Equation 90 corresponds to the charge transfer resistance for multistep single electron transfer processes in aqueous electrochemistry described in Ref. 52. However, the physical meaning behind these expressions is very different. In the aqueous electrochemical system considered in Ref. 52, the overpotential acts by modifying activation barriers for a single electron transfer and also by changing intermediate species concentrations, whereas in our model of MIEC|gas interfaces, the overpotential acts only by changing defect concentrations.

Equation 90, however, also shows that that the oxygen exchange current density cannot simply be deduced from the oxygen exchange resistance measured by impedance spectroscopy $R^{\mathrm{eq}}$. Rather, knowledge of $n$ and thus of the mechanism (atomic or molecular oxygen species in the rate limiting step) is required. Conversely, accurate extrapolation of $j^{0}$ from Tafel plots and comparison with measured $R^{\mathrm{eq}}$ from impedance spectra can allow mechanistic conclusions by determining $n$. Moreover, relation 90 questions the simple approach often used for comparing resistances of impedance spectra with tracer exchange data obtained on the same material. ${ }^{36}$ There, the oxygen exchange coefficient $k^{*}$ is a measure of the oxygen exchange current density $j^{0}$ with

$$
j^{0}=2 e k^{*} c_{\mathrm{O}}
$$

and $c_{\mathrm{O}}$ denoting the oxygen site concentration. A correct calculation of the corresponding exchange resistance $R^{*}$ (for the sake of comparison with the electrically measured $R^{\mathrm{eq}}$ ) should thus use the equation

$$
R^{*}=\frac{1}{2 n} \frac{k T}{4 e^{2} k^{*} c_{\mathrm{O}}} .
$$

The factor of $2 n$ ( 1 or 2$)$ is usually neglected so far, which is problematic for molecular oxygen in the rate determining step.

\section{Conclusions}

The kinetics of the oxygen exchange reactions are quantified by rate equations including the relevant acting species, i.e. adsorbates (or the gas phase determining the adsorbates) and defects involved in oxygen reduction or oxygen evolution. Any change of surface potential is neglected, hence reaction rates are purely defect controlled. The defect concentrations are defined by $\mu_{\mathrm{O}_{2}}$, which itself depends on $p_{\mathrm{O}_{2}}$ and overpotential, provided that the oxygen surface exchange reaction limits the overall reaction rate. The forward and backward rates, i.e. anodic and cathodic currents thus depend on the overpotential which varies defect concentrations. The dependence of the defect concentrations on overpotential (and $p_{\mathrm{O}_{2}}$ ) is given by the Brouwer diagram. Specific $p_{\mathrm{O}_{2}}$ and $\eta$ dependencies of the anodic, cathodic and net current densities can be calculated for a given rate equation with reaction orders for defects and $p_{\mathrm{O}_{2}}$ (via adsorbates) and a given Brouwer diagram. This is exemplified for LSF and two reaction mechanisms, one with atomic oxygen in the rate determining step and one with molecular oxygen species. Also, adsorption site restriction is considered in separate calculations.

These calculations revealed that frequently exponential $j-\eta$ curves result, with Tafel slopes given by three factors, one for the reaction order(s) of the defects in the rate equation, one from the slope of defect concentrations in the Brouwer diagram, and one from the Nernst relation between $\eta$ and $\mu_{\mathrm{O}_{2}}$. Hence, the slope does not reveal the number of electrons in the rate determining step. If the relevant defect concentrations are constant within the considered range of the Brouwer diagram, a Tafel slope of 0 and thus limiting currents result, despite absence of transport limitation. Since the gas $p_{\mathrm{O}_{2}}$ not only modifies adsorbate concentrations but also defect concentrations, the exponents $q$ of empirical power laws $j \propto p_{\mathrm{O}_{2}}{ }^{q}$ have often non-trivial meanings. They depend on the species in the rate limiting step (atomic or molecular oxygen) but also on the reaction orders of defects and on their $p_{\mathrm{O}_{2}}$ slope in the Brouwer diagram. For example, in the molecular mechanism considered, the oxygen exchange current density scales with $p_{\mathrm{O}_{2}}{ }^{0.75}$ to $p_{\mathrm{O}_{2}}{ }^{0.5}$, depending on $p_{\mathrm{O}_{2}}$. For the atomic mechanism $p_{\mathrm{O}_{2}}{ }^{0.5}$ to $p_{\mathrm{O}_{2}}{ }^{0.0}$ was found.

General equations for the partial pressure and overpotential dependencies are derived for anodic and cathodic currents. Those show that for a given overpotential both anodic and cathodic currents show 
the same $p_{\mathrm{O}_{2}}$ dependency. The Tafel slopes, on the other hand, differ by $4 n e / k T$ and measured slope differences can thus give valuable information on the mechanism $(n=1$ or $n=1 / 2$ indicating molecular or atomic oxygen in the rate determining step). Finally, a relation is deduced between the slope of a current-voltage curve close to equilibrium, e.g. measured by impedance spectroscopy, and the exchange current density. It is shown that knowledge of both values can also give mechanistic information on molecular or atomic oxygen species. Moreover, an inconsistency in the standard procedure of comparing tracer exchange coefficients and electrical surface exchange resistances was identified.

\section{Acknowledgment}

The authors gratefully acknowledge funding by Austrian Science Fund (FWF) project P4509-N16 and W1243-N16. This project has received funding from the European Union's Horizon 2020 research and innovation program under grant agreement No 824072 (Harvestore). There are no conflicts of interest to declare.

\section{Appendix A: Derivation of the Equilibrium Exchange Resistance}

Assuming $\Delta x=0$ and neglecting again $\mathrm{O}_{\mathrm{O}}^{\times}$and $\mathrm{Fe}_{\mathrm{Fe}}^{\times}$as defect species, we can rewrite Equation 9 explicitly with oxygen vacancies, electron holes and electrons as relevant defect species:

$$
j=j^{0}\left[\left(\frac{c_{\mathrm{V}}}{c_{\mathrm{V}}^{\mathrm{eq}}}\right)^{\nu_{\mathrm{V}, \mathrm{a}}}\left(\frac{c_{\mathrm{h}}}{c_{\mathrm{h}}^{\mathrm{eq}}}\right)^{v_{\mathrm{h}, \mathrm{a}}}\left(\frac{c_{\mathrm{e}}}{c_{\mathrm{e}}^{\mathrm{eq}}}\right)^{\nu_{\mathrm{e}, \mathrm{a}}}-\left(\frac{c_{\mathrm{V}}}{c_{\mathrm{V}}^{\mathrm{eq}}}\right)^{v_{\mathrm{V}, \mathrm{c}}}\left(\frac{c_{\mathrm{h}}}{c_{\mathrm{h}}^{\mathrm{eq}}}\right)^{v_{\mathrm{h}, \mathrm{c}}}\left(\frac{c_{\mathrm{e}}}{c_{\mathrm{e}}^{\mathrm{eq}}}\right)^{\nu_{\mathrm{e}, \mathrm{c}}}\right] .
$$

Taking the the derivative of the current density with respect to the overpotential gives

$$
\begin{aligned}
& \frac{1}{R}=\frac{\mathrm{d} j}{\mathrm{~d} \eta}=\frac{\mathrm{d}}{\mathrm{d} \eta}\left[j^{0}\left[\left(\frac{c_{\mathrm{V}}}{c_{\mathrm{V}}^{\mathrm{eq}}}\right)^{\nu_{\mathrm{V}, \mathrm{a}}}\left(\frac{c_{\mathrm{h}}}{c_{\mathrm{h}}^{\mathrm{eq}}}\right)^{\nu_{\mathrm{h}, \mathrm{a}}}\left(\frac{c_{\mathrm{e}}}{c_{\mathrm{e}}^{\mathrm{eq}}}\right)^{\nu_{\mathrm{e}, \mathrm{a}}}-\left(\frac{c_{\mathrm{V}}}{c_{\mathrm{V}}^{\mathrm{eq}}}\right)^{\nu_{\mathrm{V}, \mathrm{c}}}\left(\frac{c_{\mathrm{h}}}{c_{\mathrm{h}}^{\mathrm{eq}}}\right)^{\nu_{\mathrm{h}, \mathrm{c}}}\left(\frac{c_{\mathrm{e}}}{c_{\mathrm{e}}^{\mathrm{eq}}}\right)^{\nu_{\mathrm{e}, \mathrm{c}}}\right]\right] \\
& =j^{0}\left[\left(\frac{c_{\mathrm{h}}}{c_{\mathrm{h}}^{\mathrm{eq}}}\right)^{\nu_{\mathrm{h}, \mathrm{a}}}\left(\frac{c_{\mathrm{e}}}{c_{\mathrm{e}}^{\mathrm{eq}}}\right)^{\nu_{\mathrm{e}, \mathrm{a}}}\left(\frac{c_{\mathrm{V}}}{c_{\mathrm{V}}^{\mathrm{eq}}}\right)^{\nu_{\mathrm{V}, \mathrm{a}}-1} \nu_{\mathrm{V}, \mathrm{a}} \frac{1}{c_{\mathrm{V}}^{\mathrm{eq}}} \frac{\mathrm{d} c_{\mathrm{V}}}{\mathrm{d} \eta}\right. \\
& +\left(\frac{c_{\mathrm{V}}}{c_{\mathrm{V}}^{\mathrm{eq}}}\right)^{v_{\mathrm{V}, \mathrm{a}}}\left(\frac{c_{\mathrm{e}}}{c_{\mathrm{e}}^{\mathrm{eq}}}\right)^{v_{\mathrm{e}, \mathrm{a}}}\left(\frac{c_{\mathrm{h}}}{c_{\mathrm{h}}^{\mathrm{eq}}}\right)^{v_{\mathrm{h}, \mathrm{a}}-1} v_{\mathrm{h}, \mathrm{a}} \frac{1}{c_{\mathrm{h}}^{\mathrm{eq}}} \frac{\mathrm{d} c_{\mathrm{h}}}{\mathrm{d} \eta} \\
& +\left(\frac{c_{\mathrm{V}}}{c_{\mathrm{V}}^{\mathrm{eq}}}\right)^{\nu_{\mathrm{V}, \mathrm{a}}}\left(\frac{c_{\mathrm{h}}}{c_{\mathrm{h}}^{\mathrm{eq}}}\right)^{\nu_{\mathrm{h}, \mathrm{a}}}\left(\frac{c_{\mathrm{e}}}{c_{\mathrm{e}}^{\mathrm{eq}}}\right)^{\nu_{\mathrm{e}, \mathrm{a}}-1} \nu_{\mathrm{e}, \mathrm{a}} \frac{1}{c_{\mathrm{e}}^{\mathrm{eq}}} \frac{\mathrm{d} c_{\mathrm{e}}}{\mathrm{d} \eta} \\
& -\left(\frac{c_{\mathrm{h}}}{c_{\mathrm{h}}^{\text {eq }}}\right)^{v_{\mathrm{h}, \mathrm{c}}}\left(\frac{c_{\mathrm{e}}}{c_{\mathrm{e}}^{\text {eq }}}\right)^{v_{\mathrm{e}, \mathrm{c}}}\left(\frac{c_{\mathrm{V}}}{c_{\mathrm{V}}^{\mathrm{eq}}}\right)^{\nu_{\mathrm{V}, \mathrm{c}}-1} v_{\mathrm{V}, \mathrm{c}} \frac{1}{c_{\mathrm{V}}^{\mathrm{eq}}} \frac{\mathrm{d} c_{\mathrm{V}}}{\mathrm{d} \eta} \\
& -\left(\frac{c_{\mathrm{V}}}{c_{\mathrm{V}}^{\text {eq }}}\right)^{v_{\mathrm{V}, \mathrm{c}}}\left(\frac{c_{\mathrm{e}}}{c_{\mathrm{e}}^{\mathrm{eq}}}\right)^{v_{\mathrm{e}, \mathrm{c}}}\left(\frac{c_{\mathrm{h}}}{c_{\mathrm{h}}^{\mathrm{eq}}}\right)^{v_{\mathrm{h}, \mathrm{c}}-1} v_{\mathrm{h}, \mathrm{c}} \frac{1}{c_{\mathrm{h}}^{\mathrm{eq}}} \frac{\mathrm{d} c_{\mathrm{h}}}{\mathrm{d} \eta} \\
& \left.-\left(\frac{c_{\mathrm{V}}}{c_{\mathrm{V}}^{\mathrm{eq}}}\right)^{\nu_{\mathrm{V}, \mathrm{c}}}\left(\frac{c_{\mathrm{h}}}{c_{\mathrm{h}}^{\mathrm{eq}}}\right)^{\nu_{\mathrm{h}, \mathrm{c}}}\left(\frac{c_{\mathrm{e}}}{c_{\mathrm{e}}^{\mathrm{eq}}}\right)^{\nu_{\mathrm{e}, \mathrm{c}}-1} \nu_{\mathrm{e}, \mathrm{c}} \frac{1}{c_{\mathrm{e}}^{\mathrm{eq}}} \frac{\mathrm{d} c_{\mathrm{e}}}{\mathrm{d} \eta}\right] \text {. }
\end{aligned}
$$

Since for any defect D

$$
\frac{\mathrm{d} c_{\mathrm{D}}}{\mathrm{d} \eta}=c_{\mathrm{D}} \frac{\mathrm{d} \ln c_{\mathrm{D}}}{\mathrm{d} \eta}=c_{\mathrm{D}} \frac{\mathrm{d} \ln c_{\mathrm{D}}}{\mathrm{d} \mu_{\mathrm{O}_{2}}} \frac{\mathrm{d} \mu_{\mathrm{O}_{2}}}{\mathrm{~d} \eta}=4 e c_{\mathrm{D}} \frac{\mathrm{d} \ln c_{\mathrm{D}}}{\mathrm{d} \mu_{\mathrm{O}_{2}}},
$$

Equation A2 can be simplified to

$$
\begin{aligned}
\frac{1}{R}= & 4 e j^{0}\left[\left(\frac{c_{\mathrm{V}}}{c_{\mathrm{V}}^{\mathrm{eq}}}\right)^{v_{\mathrm{V}, \mathrm{a}}}\left(\frac{c_{\mathrm{h}}}{c_{\mathrm{h}}^{\mathrm{eq}}}\right)^{v_{\mathrm{h}, \mathrm{a}}}\left(\frac{c_{\mathrm{e}}}{c_{\mathrm{e}}^{\mathrm{eq}}}\right)^{v_{\mathrm{e}, \mathrm{a}}}\left(v_{\mathrm{V}, \mathrm{a}} \frac{\mathrm{d} \ln c_{\mathrm{V}}}{\mathrm{d} \mu_{\mathrm{O}_{2}}}+v_{\mathrm{h}, \mathrm{a}} \frac{\mathrm{d} \ln c_{\mathrm{h}}}{\mathrm{d} \mu_{\mathrm{O}_{2}}}+v_{\mathrm{e}, \mathrm{a}} \frac{\mathrm{d} \ln c_{\mathrm{e}}}{\mathrm{d} \mu_{\mathrm{O}_{2}}}\right)\right. \\
& \left.-\left(\frac{c_{\mathrm{V}}}{c_{\mathrm{V}}^{\mathrm{eq}}}\right)^{v_{\mathrm{V}, \mathrm{c}}}\left(\frac{c_{\mathrm{h}}}{c_{\mathrm{h}}^{\mathrm{eq}}}\right)^{v_{\mathrm{h}, \mathrm{c}}}\left(\frac{c_{\mathrm{e}}}{c_{\mathrm{e}}^{\mathrm{eq}}}\right)^{v_{\mathrm{e}, \mathrm{c}}}\left(v_{\mathrm{V}, \mathrm{c}} \frac{\mathrm{d} \ln c_{\mathrm{V}}}{\mathrm{d} \mu_{\mathrm{O}_{2}}}+v_{\mathrm{h}, \mathrm{c}} \frac{\mathrm{d} \ln c_{\mathrm{h}}}{\mathrm{d} \mu_{\mathrm{O}_{2}}}+v_{\mathrm{e}, \mathrm{c}} \frac{\mathrm{d} \ln c_{\mathrm{e}}}{\mathrm{d} \mu_{\mathrm{O}_{2}}}\right)\right] .
\end{aligned}
$$

Combining Equations A4, 78 and 79 gives

$$
\begin{aligned}
\frac{1}{R}= & 4 e j^{0}\left[\left(\frac{c_{\mathrm{V}}}{c_{\mathrm{V}}^{\mathrm{eq}}}\right)^{v_{\mathrm{V}, \mathrm{a}}}\left(\frac{c_{\mathrm{h}}}{c_{\mathrm{h}}^{\mathrm{eq}}}\right)^{v_{\mathrm{h}, \mathrm{a}}}\left(\frac{c_{\mathrm{e}}}{c_{\mathrm{e}}^{\mathrm{eq}}}\right)^{v_{\mathrm{e}, \mathrm{a}}}\left(\left(2 v_{\mathrm{V}, \mathrm{a}}+v_{\mathrm{h}, \mathrm{a}}-v_{\mathrm{e}, \mathrm{a}}\right) \frac{\mathrm{d} \ln c_{\mathrm{h}}}{\mathrm{d} \mu_{\mathrm{O}_{2}}}-\frac{\nu_{\mathrm{V}, \mathrm{a}}}{2 k T}\right)\right. \\
& \left.-\left(\frac{c_{\mathrm{V}}}{c_{\mathrm{V}}^{\mathrm{eq}}}\right)^{v_{\mathrm{V}, \mathrm{c}}}\left(\frac{c_{\mathrm{h}}}{c_{\mathrm{h}}^{\mathrm{eq}}}\right)^{v_{\mathrm{h}, \mathrm{c}}}\left(\frac{c_{\mathrm{e}}}{c_{\mathrm{e}}^{\mathrm{eq}}}\right)^{v_{\mathrm{e}, \mathrm{c}}}\left(\left(2 v_{\mathrm{V}, \mathrm{c}}+v_{\mathrm{h}, \mathrm{c}}-v_{\mathrm{e}, \mathrm{c}}\right) \frac{\mathrm{d} \ln c_{\mathrm{h}}}{\mathrm{d} \mu_{\mathrm{O}_{2}}}-\frac{\nu_{\mathrm{V}, \mathrm{c}}}{2 k T}\right)\right] .
\end{aligned}
$$

Including the relations between anodic and cathodic reaction orders (Equations 73 and 74) yields

$$
\begin{aligned}
\frac{1}{R}= & 4 e j^{0}\left[\left(\frac{c_{\mathrm{V}}}{c_{\mathrm{V}}^{\mathrm{eq}}}\right)^{v_{\mathrm{V}, \mathrm{a}}}\left(\frac{c_{\mathrm{h}}}{c_{\mathrm{h}}^{\mathrm{eq}}}\right)^{v_{\mathrm{h}, \mathrm{a}}}\left(\frac{c_{\mathrm{e}}}{c_{\mathrm{e}}^{\mathrm{eq}}}\right)^{v_{\mathrm{e}, \mathrm{a}}}\left(\left(2 v_{\mathrm{V}, \mathrm{a}}+v_{\mathrm{h}, \mathrm{a}}-v_{\mathrm{e}, \mathrm{a}}\right) \frac{\mathrm{d} \ln c_{\mathrm{h}}}{\mathrm{d} \mu_{\mathrm{O}_{2}}}-\frac{v_{\mathrm{V}, \mathrm{a}}}{2 k T}\right)\right. \\
& \left.-\left(\frac{c_{\mathrm{V}}}{c_{\mathrm{V}}^{\mathrm{eq}}}\right)^{v_{\mathrm{V}, \mathrm{c}}}\left(\frac{c_{\mathrm{h}}}{c_{\mathrm{h}}^{\mathrm{eq}}}\right)^{v_{\mathrm{h}, \mathrm{c}}}\left(\frac{c_{\mathrm{e}}}{c_{\mathrm{e}}^{\mathrm{eq}}}\right)^{v_{\mathrm{e}, \mathrm{c}}}\left(\left(2 v_{\mathrm{V}, \mathrm{a}}+v_{\mathrm{h}, \mathrm{a}}-v_{\mathrm{e}, \mathrm{a}}\right) \frac{\mathrm{d} \ln c_{\mathrm{h}}}{\mathrm{d}_{\mathrm{O}_{2}}}-\frac{\nu_{\mathrm{V}, \mathrm{a}}+2 n}{2 k T}\right)\right]
\end{aligned}
$$

At zero overpotential the defect concentrations are equal to their equilibrium values and thus

$$
\begin{aligned}
\frac{1}{R^{e q}} & =4 e j^{0}\left[\left(2 v_{\mathrm{V}, \mathrm{a}}+v_{\mathrm{h}, \mathrm{a}}-v_{\mathrm{e}, \mathrm{a}}\right) \frac{\mathrm{d} \ln c_{\mathrm{h}}}{\mathrm{d} \mu_{\mathrm{O}_{2}}}-\frac{v_{\mathrm{V}, \mathrm{a}}}{2 k T}-\left(2 \nu_{\mathrm{V}, \mathrm{a}}+v_{\mathrm{h}, \mathrm{a}}-v_{\mathrm{e}, \mathrm{a}}\right) \frac{\mathrm{d} \ln c_{\mathrm{h}}}{\mathrm{d} \mathrm{O}_{2}}+\frac{\nu_{\mathrm{V}, \mathrm{a}}+2 n}{2 k T}\right] \\
& =\frac{4 n e j^{0}}{k T} .
\end{aligned}
$$

\section{List of Symbols}

Defects and Adsorbates

Symbol Description

$\mathrm{V}_{\mathrm{O}} \quad$ oxygen vacancy

$\mathrm{h}^{\circ} \quad$ electron hole

$\mathrm{e}^{\prime} \quad$ electron

$\mathrm{O}_{\mathrm{O}}^{\times} \quad$ lattice oxygen

$\mathrm{Fe}_{\mathrm{Fe}}^{\times} \quad$ lattice iron

$\mathrm{O}_{\mathrm{O}}^{\cdot} \quad$ onefold positively charged oxygen

$\mathrm{O}_{\mathrm{O}}^{\bullet} \quad$ twofold positively charged oxygen

$\operatorname{ad}_{(a t)} \quad$ free adsorption site for atomic adsorbates

$\mathrm{O}_{(\mathrm{ad})} \quad$ neutral atomic adsorbate

$\mathrm{O}^{-}$(ad) negatively charged atomic adsorbate

$\mathrm{ad}_{(\mathrm{mo})} \quad$ free adsorption site for molecular adsorbates

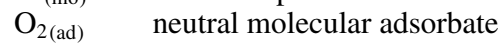

$\mathrm{O}_{2}^{-}$(ad) negatively charged molecular adsorbate

\section{Main Symbols}

Symbol Description

$e \quad$ elementary charge

$k$

$T$

$\eta$

$p_{\mathrm{O}_{2}}$

$\mu_{\mathrm{O}_{2}}$

$x$

$\chi_{0}$

$\Delta x$

$\beta$

$j$

$j^{0}$

$R$

$c$

$\theta$

$v$

$k$

K

Boltzmann's constant

absolute temperature

overpotential

oxygen partial pressure

oxygen chemical potential vs. oxygen at 1 bar surface potential

equilibrium surface potential

change of surface potential under current

surface potential dependency factor

current density

exchange current density

oxygen exchange resistance

concentration

surface coverage

reaction order

rate constant

equilibrium constant

\section{Indices}

Index

a

c

eq

D

$\mathrm{p}$

V

h

e

$\mathrm{Ox}$

$\mathrm{Fe}$

Op

Opp

(ad)

at

mo
Description

regarding the anodic direction

regarding the cathodic direction

in equilibrium

of defects (generic)

$\mathrm{p} \&$ of $p_{\mathrm{O}_{2}}$

of oxygen vacancies $\mathrm{V}_{\mathrm{O}}^{*}$

of electron holes $h^{\circ}$

of electrons $\mathrm{e}^{\prime}$

of lattice oxygen $\mathrm{O}_{\mathrm{O}}^{\times}$

of lattice iron $\mathrm{Fe}_{\mathrm{Fe}}^{\times}$

of onefold positively charged oxygen $\mathrm{O}_{\mathrm{O}}^{*}$ of twofold positively charged oxygen $\mathrm{O}_{\mathrm{O}}^{*}$ adsorbed

atomic (adsorbate)

molecular (adsorbate) 


\section{References}

1. A. Jun, J. Kim, J. Shin, and G. Kim, ChemElectroChem, 3, 511 (2016).

2. M. A. Laguna-Bercero, J. Power Sources, 203, 4 (2012).

3. N. Mahato, A. Banerjee, A. Gupta, S. Omar, and K. Balani, Prog. Mater. Sci., 72, 141 (2015).

4. C. Sun and U. Stimming, J. Power Sources, 171, 247 (2007).

5. M. M. Kuklja, E. a. Kotomin, R. Merkle, Y. a. Mastrikov, and J. Maier, Phys. Chem. Chem. Phys., 15, 5443 (2013).

6. N. Q. Minh, J. Am. Ceram. Soc., 76, 563 (1993).

7. J. M. Ralph, C. Rossignol, and R. Kumar, J. Electrochem. Soc., 150, A1518 (2003).

8. A. Stambouli and E. Traversa, Renewable and Sustainable Energy Reviews, 6, 433 (2002).

9. Y. Zheng, J. Wang, B. Yu, W. Zhang, J. Chen, J. Qiao, and J. Zhang, Chem. Soc. Rev. 46, 1427 (2017).

10. M. Ni, M. K. Leung, and D. Y. Leung, International Journal of Hydrogen Energy, 33, 2337 (2008)

11. A. K. Opitz, A. Nenning, C. Rameshan, R. Rameshan, R. Blume, M. Hävecker, A. Knop-Gericke, G. Rupprechter, J. Fleig, and B. Klötzer, Angew. Chemie - Int. Ed., 54, 2628 (2015).

12. R. De Souza, Solid State Ionics, 106, 175 (1998).

13. X. J. Chen, K. A. Khor, and S. H. Chan, Solid State Ionics, 167, 379 (2004).

14. J. Fleig, H. R. Kim, J. Jamnik, and J. Maier, Fuel Cells, 8, 330 (2008).

15. Y. A. Mastrikov, R. Merkle, E. Heifets, E. A. Kotomin, and J. Maier, J. Phys. Chem. $C, \mathbf{1 1 4}, 3017$ (2010)

16. F. S. Baumann, J. Maier, and J. Fleig, Solid State Ionics, 179, 1198 (2008).

17. F. S. Baumann, J. Fleig, M. Konuma, U. Starke, H.-U. Habermeier, and J. Maier, J. Electrochem. Soc., 152, A2074 (2005).

18. F. S. Baumann, J. Fleig, H. U. Habermeier, and J. Maier, Solid State Ionics, 177, 1071 (2006).

19. E. V. Bongio, H. Black, F. C. Raszewski, D. Edwards, C. J. Mcconville, and V. R. W. Amarakoon, Fuel Cells, 14, 193 (2005).

20. S. Kogler, A. Nenning, G. M. Rupp, A. K. Opitz, and J. Fleig, J. Electrochem. Soc., 162, F317 (2015).

21. M. Kubicek, T. M. Huber, A. Welzl, A. Penn, G. M. Rupp, J. Bernardi, M. Stöger-Pollach, H. Hutter, and J. Fleig, Solid State Ionics, 256, 38 (2014).

22. J. Mirzababaei and S. Chuang, Catalysts, 4, 146 (2014).

23. A. Nenning, A. K. Opitz, C. Rameshan, R. Rameshan, R. Blume, M. Hävecker, A. Knop-Gericke, G. Rupprechter, B. Klötzer, and J. Fleig, J. Phys. Chem. C, 120 1461 (2016).

24. G. M. Rupp, H. Téllez, J. Druce, A. Limbeck, T. Ishihara, J. Kilner, and J. Fleig, J. Mater. Chem. A, 3, 22759 (2015).

25. G. M. Rupp, A. Schmid, A. Nenning, and J. Fleig, J. Electrochem. Soc., 163, F564 (2016).

26. M. Sase, J. Suzuki, K. Yashiro, T. Otake, A. Kaimai, T. Kawada, J. Mizusaki, and H. Yugami, Solid State Ionics, 177, 1961 (2006).

27. J. E. ten Elshof, J. Electrochem. Soc., 144, 1060 (1997).

28. H. Wang, K. J. Yakal-Kremski, T. Yeh, G. M. Rupp, A. Limbeck, U. Fleig, and S. A. Barnett, J. Electrochem. Soc., 163, 581 (2016).

29. J. W. Han and B. Yildiz, Energy \& Environmental Science, 5, 8598 (2012).

30. Z. Cai, M. Kubicek, J. Fleig, and B. Yildiz, Chemistry of Materials, 24, 1116 (2012).

31. N. H. Perry, D. Pergolesi, K. Sasaki, S. R. Bishop, and H. L. Tuller, ECS Transactions, 57, 1719 (2013).
32. N. H. Perry, D. Pergolesi, S. R. Bishop, and H. L. Tuller, Solid State Ionics, 273, 18 (2015).

33. A. Staykov, H. Tellez, J. Druce, J. Wu, T. Ishihara, and J. Kilner, Science and Technology of Advanced Materials, 19, 221 (2018).

34. A. Yan, M. Cheng, Y. Dong, W. Yang, V. Maragou, S. Song, and P. Tsiakaras, Appl. Catal. B Environ., 66, 64 (2006).

35. J. F. Vente, S. McIntosh, W. G. Haije, and H. J. M. Bouwmeester, J. Solid State Electrochem., 10, 581 (2006).

36. F. S. Baumann, J. Fleig, H. U. Habermeier, and J. Maier, Solid State Ionics, 177, 3187 (2006).

37. T. Kawada, J. Suzuki, M. Sase, A. Kaimai, K. Yashiro, Y. Nigara, J. Mizusaki, K. Kawamura, and H. Yugami, J. Electrochem. Soc., 149, E252 (2002).

38. J. A. Kilner, R. A. De Souza, and I. C. Fullarton, Solid State Ionics, 86-88, 703 (1996).

39. J. Yoo, A. Verma, S. Wang, and A. J. Jacobson, J. Electrochem. Soc., 152, A497 (2005).

40. L. Wang, R. Merkle, and J. Maier, J. Electrochem. Soc., 157, B1802 (2010).

41. J. Fleig, R. Merkle, J. Maier, J. Maier, J. Maier, M. Che, A. J. Tench, M. Che, A. J. Tench, Y. Wang, D. Pillay, G. S. Hwang, Y. D. Kim, A. P. Seitsonen, S. Wendt, J. Wang, C. Fan, K. Jacobi, H. Over, G. Ertl, P. B. Weisz, H. Geistlinger, D. Kohl, A. Gurlo, A. Rothschild, Y. Komem, N. Ashkenasy, S. B. Adler, X. Y. Chen, J. R. Wilson, J. Fleig, J. Jamnik, J. Fleig, J. Mizusaki, K. Amano, S. Yamaguchi, K. Fueki, R. A. Marcus, R. A. Marcus, H. Gerischer, E. Bucher, W. Sitte, G. B. Caraman, V. A. Cherepanov, T. V. Aksenova, and M. V. Ananyev, Phys. Chem. Chem. Phys., 9, 2713 (2007).

42. S. Adler, Solid State Ionics, 111, 125 (1998).

43. S. B. Adler, X. Y. Chen, and J. R. Wilson, J. Catal., 245, 91 (2007).

44. O. Yamamoto, Solid State Ionics, 22, 241 (1987)

45. T. Kawada, K. Masuda, and J. Suzuki, Solid State Ionics, 121, 271 (1999).

46. Z. Guan, D. Chen, and W. C. Chueh, Phys. Chem. Chem. Phys., 19, 23414 (2017).

47. J. Fleig, Phys. Chem. Chem. Phys., 7, 2027 (2005).

48. A. Schmid, G. M. Rupp, and J. Fleig, Chemistry of Materials, 30, 4242 (2018).

49. A. Schmid, G. M. Rupp, and J. Fleig, Physical Chemistry Chemical Physics, 20, 12016 (2018).

50. J. Fleig, G. M. Rupp, A. Nenning, and A. Schmid, ECS Trans., 77, 93 (2017).

51. J. Maier, Physical Chemistry of Ionic Materials: Ions and Electrons in Solids, Wiley, 2004.

52. A. Bard and L. Faulkner, Electrochemical Methods: Fundamentals and Applications, Wiley, 2000.

53. R. Zohourian, R. Merkle, and J. Maier, ECS Trans., 77, 133 (2017)

54. R. Zohourian, R. Merkle, G. Raimondi, and J. Maier, Advanced Functional Materials, 28, 1801241 (2018).

55. D. N. Mueller, M. L. Machala, H. Bluhm, and W. C. Chueh, Nature Communications, 6, 6097 (2015).

56. J. Mizusaki, M. Yoshihiro, S. Yamauchi, and K. Fueki, J. Solid State Chem., 58, 257 (1985).

57. M. Kuhn, S. Hashimoto, K. Sato, K. Yashiro, and J. Mizusaki, Solid State Ionics, 195, 7 (2011).

58. J. Mizusaki, M. Yoshihiro, S. Yamauchi, and K. Fueki, J. Solid State Chem., 67, 1 (1987).

59. E. Bucher and W. Sitte, J. Electroceramics, 13, 779 (2004). 